\title{
Evolution of topological defects at two sequential phase transitions of $\mathrm{Nd}_{2} \mathrm{SrFe}_{2} \mathrm{O}_{7}$
}

\author{
Fei-Ting Huang, ${ }^{1, *}$ Yanbin Li $\odot,{ }^{1,2, *}$ Fei Xue $\odot,{ }^{3, *}$ Jae-Wook Kim $\odot,{ }^{1}$ Lunyong Zhang, ${ }^{4}$ Ming-Wen Chu, ${ }^{5}$ \\ Long-Qing Chen, ${ }^{3}$ and Sang-Wook Cheong ${ }^{1, \dagger}$ \\ ${ }^{1}$ Rutgers Center for Emergent Materials and Department of Physics and Astronomy, Rutgers University, Piscataway, New Jersey 08854, USA \\ ${ }^{2}$ State Key Laboratory of Crystal Materials, Shandong University, Jinan 250100, China \\ ${ }^{3}$ Department of Materials Science and Engineering, The Pennsylvania State University, University Park, Pennsylvania 16802, USA \\ ${ }^{4}$ Laboratory for Pohang Emergent Materials and Max Plank POSTECH Center for Complex Phase Materials, \\ Pohang University of Science and Technology, Pohang 790-784, Korea \\ ${ }^{5}$ Center for Condensed Matter Sciences and Center of Atomic Initiative for New Materials, National Taiwan University, Taipei 106, Taiwan
}

(Received 8 August 2020; revised 4 March 2021; accepted 13 May 2021; published 21 June 2021)

\begin{abstract}
How topological defects, unavoidable at symmetry-breaking phase transitions in a wide range of systems, evolve through consecutive phase transitions with different broken symmetries remains unexplored. $\mathrm{Nd}_{2} \mathrm{SrFe}_{2} \mathrm{O}_{7}$, a bilayer ferrite, exhibits two intriguing structural phase transitions and dense networks of the so-called type II $Z_{8}$ structural vortices at room temperature, so it is an ideal system to explore the topological defect evolution. From our extensive experimental investigation, we demonstrate that the cooling rate at the second-order transition $\left(1290^{\circ} \mathrm{C}\right)$ plays a decisive role in determining the vortex density at room temperature, following the universal Kibble-Zurek mechanism. In addition, we discovered a transformation between topologically distinct vortices $\left(Z_{8}\right.$ to $Z_{4}$ vortices) at the first-order transition $\left(550^{\circ} \mathrm{C}\right)$, which conserves the number of vortex cores. Remarkably, the $\mathrm{Z}_{4}$ vortices consist of two phases with an identical symmetry but two distinct magnitudes of an order parameter. Furthermore, when lattice distortion is enhanced by chemical doping, an alternative type of topological defects emerges: loop domain walls with orthorhombic distortions in the tetragonal background, resulting in unique pseudo-orthorhombic twins. Our findings open an avenue to explore the evolution of topological defects through multiple phase transitions.
\end{abstract}

DOI: 10.1103/PhysRevResearch.3.023216

\section{INTRODUCTION}

Recent progress in observational cosmology is remarkable and testing the limits of our knowledge [1]. In parallel, the ground-based quantized vortices of superfluids [2,3], optical vortices of liquid crystals [4,5], multiferroic vortices [6-9], polarization screws of oxide superlattices [10,11], and magnetic skyrmions [12] in condensed matter have made compelling analogies to putative cosmological topological defects such as cosmic strings and cosmic domain walls (DWs) $[13,14]$. Understanding topological defects at phase transitions is particularly important in light of their crucial role as fingerprints of order parameter topologies $[7,15]$ and phase fluctuations at the critical temperature through the Kibble-Zurek mechanism [13,14]. The Kibble-Zurek mechanism describes the scaling of the defect density and how information spreads at a finite speed in a system that is driven through a continuous phase transition. It has been effectively

\footnotetext{
*These authors contributed equally to this work.

${ }^{\dagger}$ Corresponding author: sangc@ $@$ physics.rutgers.edu

Published by the American Physical Society under the terms of the Creative Commons Attribution 4.0 International license. Further distribution of this work must maintain attribution to the author(s) and the published article's title, journal citation, and DOI.
}

tested on a variety of condensed matter systems such as liquid crystals [16,17], superfluid ${ }^{3} \mathrm{He}[2]$, and multiferroics $[7,18]$. However, little is known about how topological defects evolve upon crossing multiple phase transitions.

The prototypical model used to describe spontaneous symmetry breaking is associated with a Mexican hat-type potential-energy landscape [19-21], where the distance from the peak and the angle around the axis represent the magnitude and phase of an order parameter, respectively. A system characterized by this model energy landscape may have a continuous $U(1)$ symmetry peak near the critical temperature $\left(T_{\mathrm{c}}\right)$, which plays a key role in the initial topological vortex-like defect formation at $T_{\mathrm{c}}$ before the system falls to the brim with discrete global minima at low temperatures, leading to discrete vortices. Multiferroic hexagonal manganite is an example of a phase transition described by such an energy landscape [19,21]. Interestingly, the restoration of a continuous $U(1)$ symmetry has been predicted and confirmed at vortex cores even at low temperatures [20,22-24], leading to topological protection from low-energy perturbations [25]. Thus, vortex domains are protected in a symmetry sense. This unique topological protection may enable a transformation between two different types of topological vortices. This possibility arises when the regions with the metastable-state symmetry being trapped within the ground-state vortex grow as the metastable state becomes favored via thermal activation, chemical doping, or mechanical means. The left path 

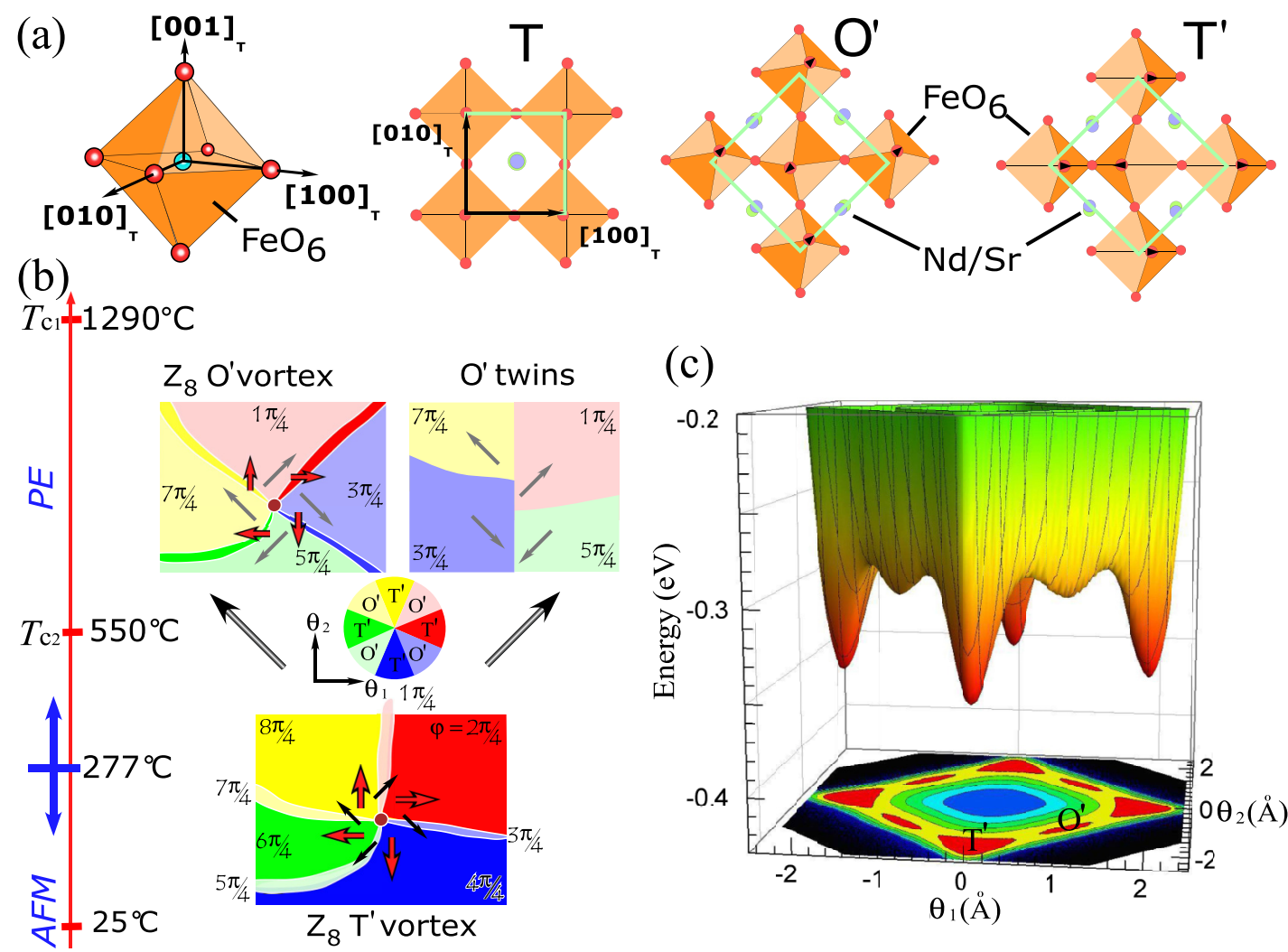

(c)

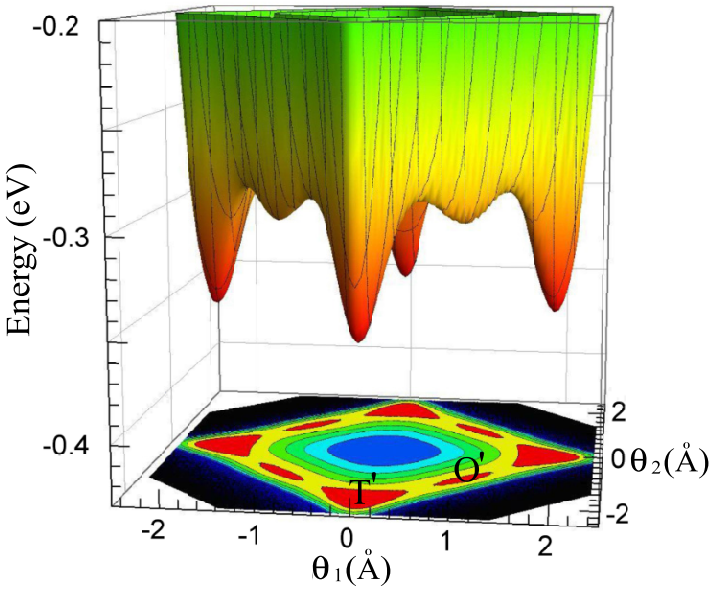

FIG. 1. (a) Two sequential phase transitions of $\mathrm{Nd}_{2} \mathrm{Ca}_{x} \mathrm{Sr}_{1-x} \mathrm{Fe}_{2} \mathrm{O}_{7}$, high-temperature $T(I 4 / m m m) \rightarrow \mathrm{O}^{\prime}($ Amam $) \rightarrow$ room-temperature $\mathrm{T}^{\prime}\left(\mathrm{P}_{2} / \mathrm{mnm}\right)$, associated with various tilts of the $\mathrm{FeO}_{6}$ octahedra. Black arrowheads indicate directions of apical oxygen (red spheres) displacements. (b) Reported $T_{\mathrm{c} 1}\left(\mathrm{~T}\right.$ to $\left.\mathrm{O}^{\prime}\right)$ and $T_{\mathrm{c} 2}\left(\mathrm{O}^{\prime}\right.$ to $\left.\mathrm{T}^{\prime}\right)$ transitions in $\mathrm{Nd}_{2} \mathrm{SrFe}_{2} \mathrm{O}_{7}$. Type $\mathrm{II} \mathrm{Z}_{8} \mathrm{~T}^{\prime}$ vortex forms at room temperature. Left path illustrates a type II $\mathrm{Z}_{8} \mathrm{~T}^{\prime}$ vortex to a type II $\mathrm{Z}_{8} \mathrm{O}^{\prime}$ vortex transformation through $\mathrm{T}^{\prime}$ domains narrowing (dark colors) and $\mathrm{O}^{\prime}$ walls broadening (light colors) across $T_{\mathrm{c} 2}$. The transformation occurs around an immobilized brown vortex core. Right path depicts the nonvortex transformation by developing orthorhombic $\mathrm{O}^{\prime}$ twins across $T_{\mathrm{c} 2}$. A color wheel illustrates the color assignment based on the order parameter direction and the corresponding phase $\varphi=\frac{n \pi}{4}(n=1-8)$. Red and black arrows denote the $\mathrm{T}^{\prime}$ and $\mathrm{O}^{\prime}$ phases, respectively. Gray arrows signify the $\mathrm{O}^{\prime}$ phase under a stimulus, and it may or may not share the same tilt magnitude as the black ones. (c) Energy landscape for the doped $\mathrm{Nd}_{2} \mathrm{SrFe}_{2} \mathrm{O}_{7}$ system, which is fitted based on the DFT calculations at $0 \mathrm{~K}$. Note that the presence of four global minima of $\mathrm{T}^{\prime}$ phase in the brim and four local minima of $\mathrm{O}^{\prime}$ phase between them. $\theta_{1}$ and $\theta_{2}$ denote the $X_{3}^{-}$mode tilt of oxygen octahedron.

in Fig. 1(b) depicts this scenario, while the right path illustrates the commonly observed $\mathrm{O}^{\prime}$ orthorhombic twins with $90^{\circ}$-type straight and $180^{\circ}$-type curved DWs. Dark and light colors correspond to the ground state and a metastable state, respectively. In the left path, the brown vortex core acts as a nucleation center, and DWs are associated with local minima, separating the global minima in an energy landscape as shown in Fig. 1(c). The vortex structures are preserved when the system switches between global minima and local minima. Thus, for example, metastable-state vortices may evolve from ground-state vortices far below the critical temperature. The first requirement to realize such vortex transformation is to find a system having DWs with metastability that can be controlled.

From our study on (Nd, $\mathrm{Tb}, \mathrm{Sr})_{3} \mathrm{Fe}_{2} \mathrm{O}_{7}, \quad n=2$ Ruddlesden-Popper (RP) ferrite, we have discovered a vortex-to-vortex transformation, which is unexpected but consistent with topological protection. Ferrite is an intriguing system that exhibits one high-temperature second-order structural transition and a following first-order structural transition above room temperature in addition to two magnetically ordered states at low temperatures [26-29], so it is an ideal system to explore topological phase transitions systematically within one system. Figures 1(a) and 1(b) illustrate the details of various relevant structures. First, we have discovered the so-called type II $Z_{8}$ structural vortices structural vortices at room temperature and examined the dynamics of $Z_{8} T^{\prime}$ structural vortices utilizing in situ heating dark-field transmission electron microscopy (DF-TEM). We have explored the effects of thermally induced kinetic processes, long-range spontaneous distortion, and chemical doping on the resulting topological defect patterns and attempted to explain the origin of domain configurations by phase-field simulations. Our results show that, despite multiple phase transitions, the Kibble-Zurek mechanism is validated if the length scale is set at a second-order critical temperature. We have also unveiled an unexpected vortex-to-loop transformation due to the enlarged elastic interaction with chemical doping and thermodynamic and strain/pressure controls of topological defect patterns. 


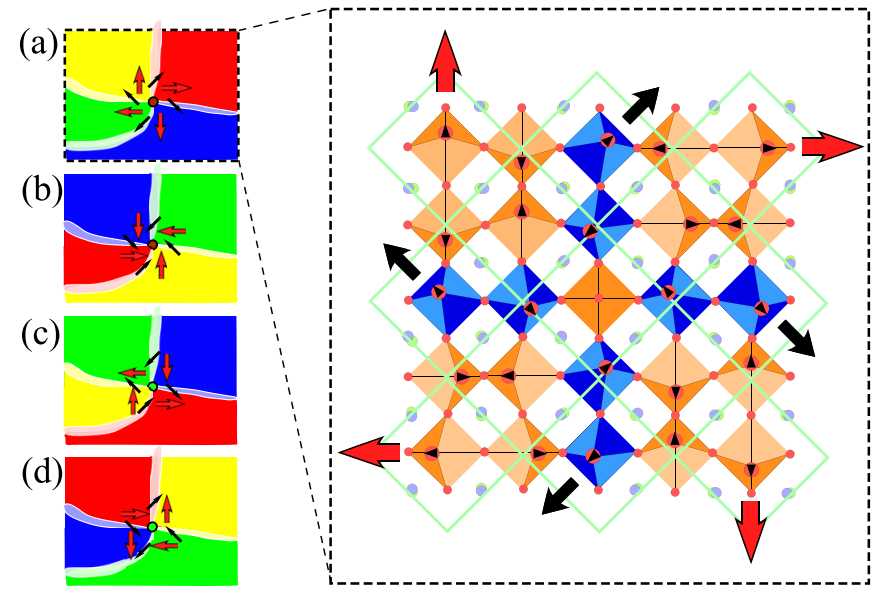

FIG. 2. (a), (b) Two types of vortex-like domains and (c), (d) two types of antivortex-like domains observed in Fig. 3(f). The schematics in the right panel illustrate the local distortion of the $Z_{8}$ vortex. Black arrowheads indicate directions of apical oxygen (red spheres) displacements. The green squares for the $\sqrt{ } 2 \times \sqrt{ } 2$ supercells demonstrate the continuous rotation with various octahedral tilts around an untilted core showing the T symmetry.

\section{POSSIBLE SCENARIOS OF VORTEX TRANSFORMATION}

Intriguing structural type II $\mathrm{Z}_{8} \mathrm{~T}^{\prime}$ structural vortices (eight-state vortex-antivortex pairs) were recently found in $\mathrm{Ca}_{2} \mathrm{SrTi}_{2} \mathrm{O}_{7}, n=2 \mathrm{RP}$ bilayered perovskite [30]. They exhibit a unique real-space topology in which domains and DWs are intricately intertwined with two sets of octahedral tilts marked by red and black arrows [the bottom cartoon of Fig. 1(b)]. Figure 2(a) illustrates those sets of arrows, rotating every $45^{\circ}$, are associated with two distinct symmetries: an orthorhombic $\mathrm{O}^{\prime}$ phase (Amam) and tetragonal $\mathrm{T}^{\prime}$ phase $\left(P 4_{2} / \mathrm{mnm}\right)$, respectively. The smooth $90^{\circ}$ rotating, for example, from an up-tilt to a right-tilt state [Fig. 2(a) yellow to red domains], occurs via a diagonal-tilt state [light-red DW in Fig. 2(a)], resulting in a sequence of all consecutive eight states around anun-tilted core in brown [Fig. 2(a)]. Note that four states of the $\mathrm{O}^{\prime}$ phase appear narrow as DWs and the other four states of the $\mathrm{T}^{\prime}$ phase remain broad as domains. This type of domain configuration with large disparity in size is called type II domains, and, otherwise, type I. We emphasize that the symmetry in the order parameter space is fourfold, but four distinct domain states and four distinct domain wall states are present around a vortex core, and an oxygen displacement vector relevant to the order parameter rotates eight times around vortex core, so we call these vortices type II $\mathrm{Z}_{8}$ vortices. Experimentally, however, $\mathrm{Ca}_{2} \mathrm{SrTi}_{2} \mathrm{O}_{7}$ reveals a direct tetragonal $\mathrm{T}$ to $\mathrm{T}^{\prime}$ structural transition, indicating a significant instability of the desired orthorhombic $\mathrm{O}^{\prime}$ phase [30].

On the other hand, $\mathrm{Nd}_{2} \mathrm{SrFe}_{2} \mathrm{O}_{7}$ ferrite, the structural analogy of titanates, was reported to show two consecutive structural transitions with the target orthorhombic $\mathrm{O}^{\prime}$ phase as an intermediate symmetry appearing above $550^{\circ} \mathrm{C}[26,31]$. It first undergoes a second-order structural transition from the high-temperature $\mathrm{T}$ phase to the intermediate orthorhombic $\mathrm{O}^{\prime}$ phase, denoted as $\mathrm{H}-\mathrm{T} / \mathrm{O}^{\prime}$ transition, at the critical temperature
$T_{\mathrm{c} 1}$ of $\sim 1290^{\circ} \mathrm{C}$. A following first-order structural transition, ${\mathrm{L}-\mathrm{O}^{\prime}}^{\prime} \mathrm{T}^{\prime}$ transition denoted as $T_{\mathrm{c} 2}$, occurs from the $\mathrm{O}^{\prime}$ phase to the ground-state $\mathrm{T}^{\prime}$ phase. Note that $\mathrm{O}^{\prime}$ symmetry is a subgroup of $\mathrm{T}$ symmetry, which is consistent with the secondorder nature across $T_{\mathrm{c} 1}$, while $\mathrm{T}^{\prime}$ symmetry is not a subgroup of $\mathrm{O}^{\prime}$ phase, which is in accordance with the first-order nature of $T_{\mathrm{c} 2}$. Microscopically, $\mathrm{O}^{\prime}$ and $\mathrm{T}^{\prime}$ phase are described by different tilts of the $\mathrm{FeO}_{6}$ octahedra [Fig. 1(a)]: the $\mathrm{T}^{\prime}$ phase has the tilt axis along the $\mathrm{Fe}-\mathrm{O}$ bond direction, defined as the $[100]_{\mathrm{T}}$, while the tilt occurs along the diagonal direction, i.e., the $[110]_{\mathrm{T}}$ in the $\mathrm{O}^{\prime}$ phase. Figure 1 (a) illustrates the in-plane structural models in which the black arrowheads show the details of apical oxygen tilts. The tilts of $\mathrm{FeO}_{6}$ octahedra result from the condensation of the $X_{3}^{-}$soft mode. Based on Landau theory, we plotted an energy landscape Fig. 1(c) as a function of order parameters with polar coordinates $(Q$, $\varphi)$. Here $Q$ and $\varphi$ are the magnitude and phase of the $X_{3}^{-}$ mode, respectively. With the in-plane axis of the $\mathrm{T}$ phase as the polar axis, the phase $\varphi$ can take the value $\frac{n \pi}{4}(n=1-8)$. When $n$ is an even number, the system is in the $\mathrm{T}^{\prime}$ phase, while an odd $n$ indicates an $\mathrm{O}^{\prime}$ phase. The $\mathrm{T}^{\prime}$ phase and $\mathrm{O}^{\prime}$ phase correspond to the global minima and local minima in the energy landscape, respectively. The energy barriers between the global minima $\left(\mathrm{T}^{\prime}\right)$, and the local minima $\left(\mathrm{O}^{\prime}\right)$ is $\sim 65 \mathrm{meV}$, which is about two times of that in hexagonal $\mathrm{YMnO}_{3}$ [19]. Full computational details [32-36] are given in Appendix A. In addition to two sequential structural transitions, the system is in-plane antiferromagnetic below 550 K [27-29], and spins are aligned along the $\mathrm{c}$ axis below $15 \mathrm{~K}$ (the details are discussed in Appendix B).

\section{THE KIBBLE-ZUREK MECHANISM}

Figures 3(a)-3(c) show our superlattice DF-TEM images in a $\mathrm{Nd}_{2} \mathrm{SrFe}_{2} \mathrm{O}_{7}$ single crystal taken under different tilting conditions and its corresponding diffraction pattern along the $[001]_{\mathrm{T}}$ direction [Fig. 3(d)]. The curved dark-contrast lines reveal boundaries of four bright $\mathrm{T}^{\prime}$-phase domains merging at one core, which is a non- $\mathrm{T}^{\prime}$ state. Intriguingly, those curved DWs exhibit two distinct extinction rules in the superlattice DF-TEM images [Figs. 3(b) and 3(c)], in which the inequivalent nature of these two types of DWs indicates an orthorhombic-like local structure at those walls, consistent with Ref. [30]. The directions of apical oxygen distortions are denoted as black arrows in the insets of Figs. 3(b) and $3(c)$ associated with $[110]_{\mathrm{T}}$ and $[110]_{\mathrm{T}}$ tilt axes, respectively. First, the domain pattern can be viewed as a cut-through graph of these two types of $\mathrm{O}^{\prime}$-symmetry DWs [light-red and light-yellow bold lines in Fig. 3(e)]. Note that the relative spontaneous distortion $\pm \mathrm{a}_{\mathrm{O}^{\prime}}$ directions can be identified from the related electron diffraction patterns [Fig. 3(d)], but the absolute distortion direction cannot be. Thus, once the distortion direction is chosen for DWs, then the distortion directions in neighboring domains can be fully assigned without ambiguity. Therefore, oxygen octahedra inside the bright contrast domains tilt along either $[100]_{\mathrm{T}}$ or $[010]_{\mathrm{T}}$ directions, whereas those in the dark-contrast DWs tilt along diagonal $\langle 110\rangle_{\mathrm{T}}$ directions. Figure 3(f) shows one possible mapping of eight variants in the domains and DWs. The vortices and antivortices are categorized based on the cycling sequence of 

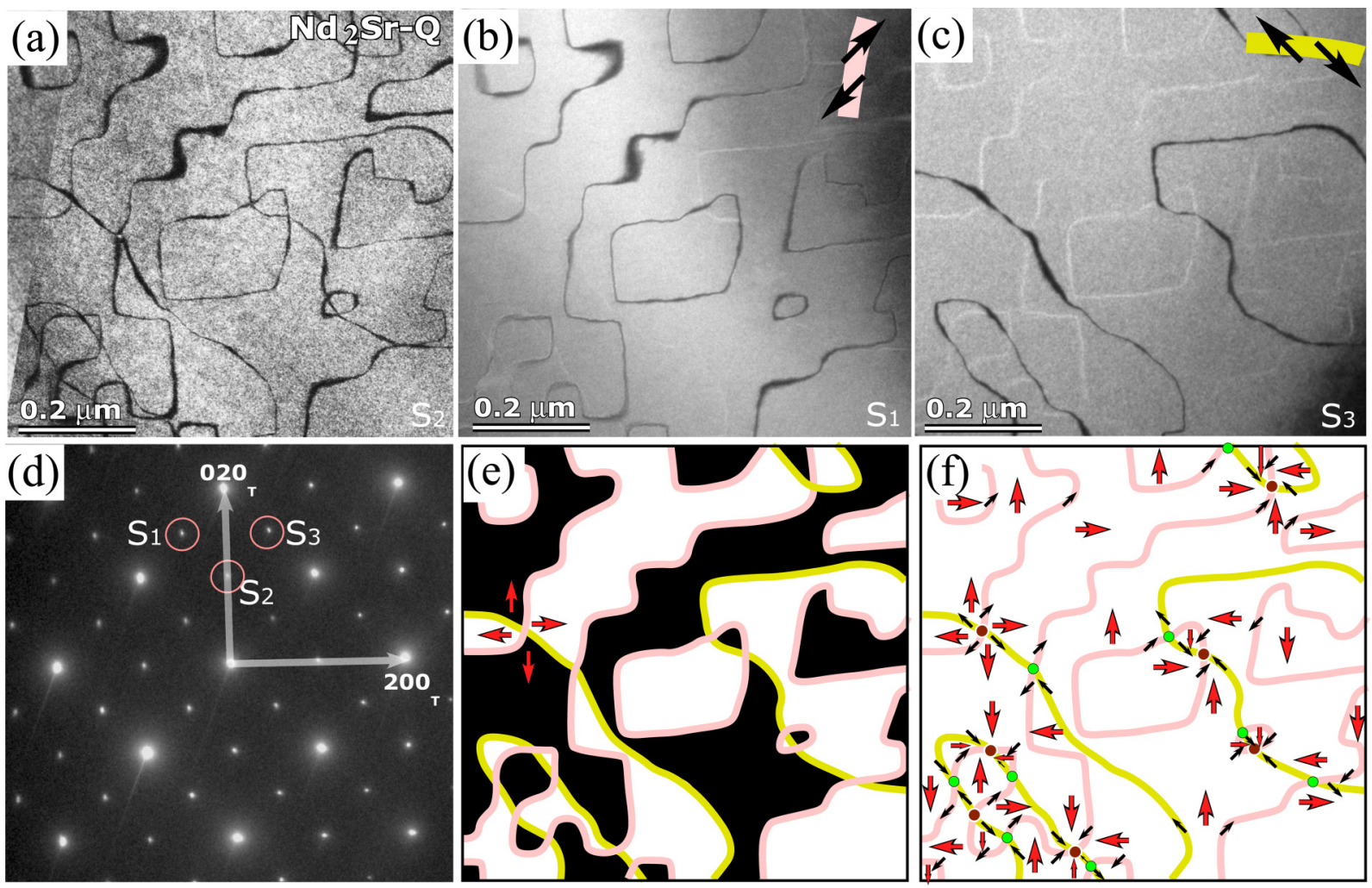

FIG. 3. DF-TEM images of $\mathrm{Nd}_{2} \mathrm{SrFe}_{2} \mathrm{O}_{7}$-Q taken under different tilting conditions, showing antiphase boundaries, which appear as dark lines with spots (a) $S_{2}=1 / 2(200)_{\mathrm{T}}=(110)_{\mathrm{T}^{\prime}}$, (b) $S_{1}=1 / 2(3 \overline{1} 0)_{\mathrm{T}}=(120)_{\mathrm{T}^{\prime}}$, and (c) $\mathrm{S}_{3}=1 / 2(310)_{\mathrm{T}}=1 / 2(210)_{\mathrm{T}^{\prime}}$. Panels $(\mathrm{b})$ and (c) reveal only a part of DWs in (a), indicating the inequivalent nature of those DWs. Octahedral tilting directions in real space are of those dark lines are shown in the upper-right corner. (d) Selected area electron diffraction (SAED) pattern along [001] projection. (e) A cut-through graph can be readily constructed. Light-yellow lines never cross any light-yellow lines, and light-red lines never cross any light-red lines. Specifically, it is a cut-through graph with two types of DWs. Two-proper coloring, black and white, is sufficient to identify the domains without the neighboring domains sharing the same color. Black and white $\mathrm{T}^{\prime}$ domains are related with $[100]_{\mathrm{T}}$ and $[010]_{\mathrm{T}}$ tilt axes (red arrows). (f) The full mapping of eight variants in the domains and DWs and the full identification of vortices (brown) and antivortices (green). A vortex is always surrounded by antivortices connected with DWs and vice versa.

the eight states around the cores. Figure 2 identifies four types of (anti-)vortex domains observed in Fig. 3(f).

We found that the vortex density D (core number/area) remains intact with various thermal treatments below $1280^{\circ} \mathrm{C}$ but changes dramatically above $1300^{\circ} \mathrm{C}$, indicating that the $T_{\mathrm{c} 1} \approx 1290^{\circ} \mathrm{C}$, rather than $T_{c 2} \approx 550^{\circ} \mathrm{C}$, transition is directly relevant to the vortex density. Figures 4(a)-4(d) show our superlattice DF-TEM images in a $\mathrm{Nd}_{2} \mathrm{SrFe}_{2} \mathrm{O}_{7}$ single crystal with various cooling rates across $T_{\mathrm{c} 1}$. The full spatial maps are shown in Appendix C. Indeed, the vortex density varies in a systematic manner, following the universal behavior of the Kibble-Zurek scaling mechanism for cooling rates $t$ across $T_{\mathrm{c} 1} \approx 1290^{\circ} \mathrm{C}$ in the range of 5 to $7000^{\circ} \mathrm{C} / \mathrm{h}$. The largest vortex density of about $21.6 \mu \mathrm{m}^{-2}$ is experimentally observed at the highest cooling rate value as shown in Fig. 4(a). The cooling-rate dependence of the vortex density follows a power law $\left\{D \propto t^{\mathrm{n}}\right\}$ with the exponent $n$ of 0.59 [Fig. 4(d) inset], reminiscent of the value for multiferroic hexagonal $\mathrm{RMnO}_{3}$ $(\mathrm{R}=$ rare earths $)$ [7].

Type II $\mathrm{Z}_{8} \mathrm{~T}^{\prime}$ (anti-)vortices span the whole bulk as observed not only in the $a b$ plane, but also in an $a c$ surface as shown in Fig. 4(e). Domain walls tend to elongate along the $c$-axis direction, probably due to the translational shift of a half $c$-lattice at DWs. An extended three-dimensional (3D) picture is illustrated in Fig. 4(f). In a two-dimensional (2D) surface [right panels in Fig. 4(f)], four domains and four DWs consisting of two types of $\mathrm{O}^{\prime}$-symmetry DWs [light-yellow and light-red curves in Fig. 4(f)] meet at a core [brown and green circles in Fig. 4(f)]. In 3D space, those cores converge to a vortex line [black line in Fig. 4(f)]. By symmetry, the tilt axes of the $\mathrm{T}^{\prime}$ domains rotate by $90^{\circ}$ in adjacent bilayers [red arrows in Fig. 4(f)], so an antivortex is always right beneath a vortex in the adjacent bilayers, and vice versa, and vortex and antivortex alternate along a vortex line.

\section{IN SITU HEATING DOMAIN OBSERVATION}

To investigate the possible vortex-to-vortex transformation that we discussed earlier, we further performed in situ TEM heating experiments above $T_{\mathrm{c} 2}$ on $\mathrm{Nd}_{2} \mathrm{SrFe}_{2} \mathrm{O}_{7}$ single crystals. Two specimens with initial low [Figs. 5(b) and 5(c)] and high [Figs. 5(e) and 5(f)] type II $\mathrm{Z}_{8} \mathrm{~T}^{\prime}$ vortex densities, resulting from different cooling rate across $T_{\mathrm{c} 1}$, were examined. Upon heating, the $S_{2}=1 / 2(200)_{\mathrm{T}}=(110)_{\mathrm{T}^{\prime}}$ spots begin to fade [Fig. 5(a)] while the $S_{1} / S_{3}$ spots become stronger over a wide temperature range, indicating the hysteretic phase transformation from the $\mathrm{T}^{\prime}$ to $\mathrm{O}_{T \mathrm{c} 2}^{\prime}$ phases. In real space, four-level colors exist; rectangular domains with dark- and 

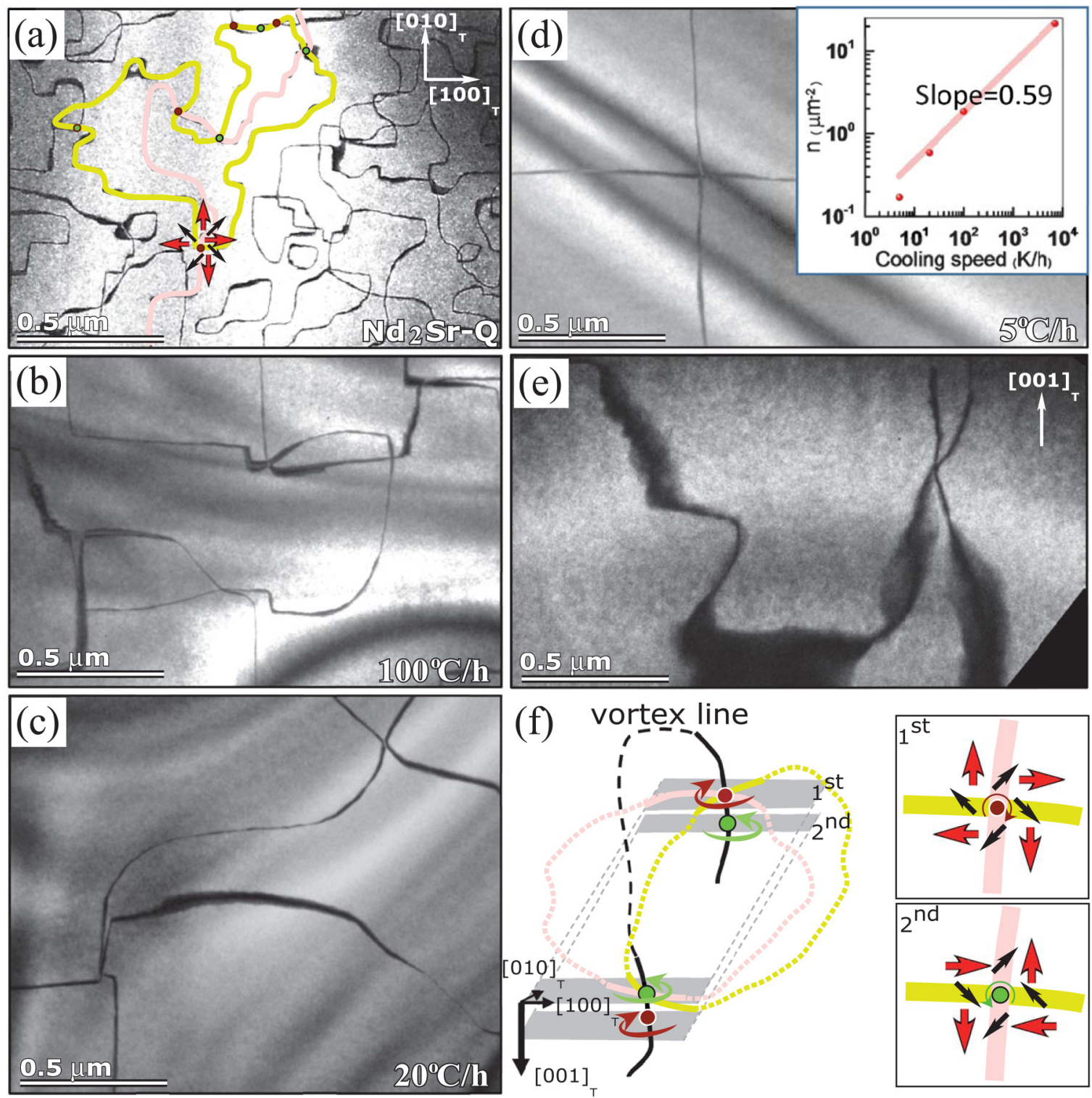

FIG. 4. Cooling rate-dependent type II $\mathrm{Z}_{8} \mathrm{~T}^{\prime}$ vortex domain patterns in $\mathrm{Nd}_{2} \mathrm{SrFe}_{2} \mathrm{O}_{7}$ crystals. Light-yellow and light-red bold lines represent DWs of the $\mathrm{O}^{\prime}$-symmetry originated from $[110]_{\mathrm{T}}$ and $[110]_{\mathrm{T}}$ tilt axes. In-plane DF-TEM images of crystals cooling from $1300^{\circ} \mathrm{C}\left(\mathrm{above} T_{\mathrm{c} 1}\right)$ by (a) fast quenching $\left(7000^{\circ} \mathrm{C} / \mathrm{h}\right.$ across $\left.T_{\mathrm{c} 1}\right)$, (b) with a cooling rate of $100^{\circ} \mathrm{C} / \mathrm{h}$, (c) $20^{\circ} \mathrm{C} / \mathrm{h}$, and (d) $5^{\circ} \mathrm{C} / \mathrm{h}$. The inset shows the cooling rate dependence of the vortex density (vortex number/area), exhibiting the Kibble-Zurek-like increase of vortex density with cooling rate. (e) Side-view DF-TEM image showing an elongated type II $Z_{8} \mathrm{~T}^{\prime}$ vortex. (f) The schematic of a 3D vortex line connecting cores of vortices (brown circles) and antivortices (green circles). Black, light-red, and light-yellow curved lines indicate vortex line and two types of DWs, respectively. The right panel illustrates the $90^{\circ}$ domain relation (red arrows) between adjacent bilayers along the $c$-direction.

light-gray contrasts appear above $T_{\mathrm{c} 2}$ [Figs. 5(b) and 5(c)], corresponding to two types of $\mathrm{O}^{\prime} T_{\mathrm{c} 2}$ orthorhombic twins in addition to the leftover $\mathrm{O}^{\prime}$-symmetry DWs with dark and bright contrasts. The reverse contrasts between Figs. 5(b) and 5(c) further confirm the $\mathrm{O}^{\prime}{ }_{T \mathrm{c} 2}$ twin nature. Analogously, the $\mathrm{O}_{T \mathrm{c} 2}^{\prime}$ orthorhombic twins form at $600^{\circ} \mathrm{C}$ in the specimen with a higher $Z_{8} T^{\prime}$ vortex density, a few hundred $n m$ in length though [Figs. 5(e) and 5(f)]. The density of $\mathrm{O}^{\prime}{ }_{T \mathrm{c} 2}$ orthorhombic twins reveals a positive correlation with the initial type II $\mathrm{Z}_{8} \mathrm{~T}^{\prime}$ vortex density, i.e., the cooling rate across $T_{\mathrm{c} 1} \approx 1290^{\circ} \mathrm{C}$.

At first sight, instead of seeing the vortex-vortex transformation [left path in Fig. 1(b)], lamellar twins appear above
$T_{c 2}$. A carefully analysis of color levels in Figs. 5(b) and 5 (c) reveals the formation of $\mathrm{Z}_{4} \mathrm{O}^{\prime}{ }^{\prime} \mathrm{c} 2$ vortices, which consist of four $\mathrm{O}^{\prime}$ states, i.e., $\varphi=\frac{n \pi}{4}(n=1,3,5,7)$ meeting at one point when considering the finite-width $\mathrm{O}^{\prime}$ DWs as narrow domains (see Appendix C). Figure 5(d) is the vector map derived from Figs. 5(b) and 5(c). For the sake of simplicity, two colors (light-red and light-yellow) instead of four colors are applied for the four states of the $\mathrm{O}^{\prime}{ }_{\mathrm{Tc} 2}$ phase. No $\mathrm{T}^{\prime}$ phase exists. The nucleated $\mathrm{O}_{T \mathrm{c} 2}^{\prime}$ phase contains a larger magnitude of the tilt component than the $\mathrm{O}^{\prime}$ phase of original DWs. The in situ DF-TEM analysis and structural factors with different octahedral tilting amplitudes are shown in Appendix C. The same extinction condition and the weaker 

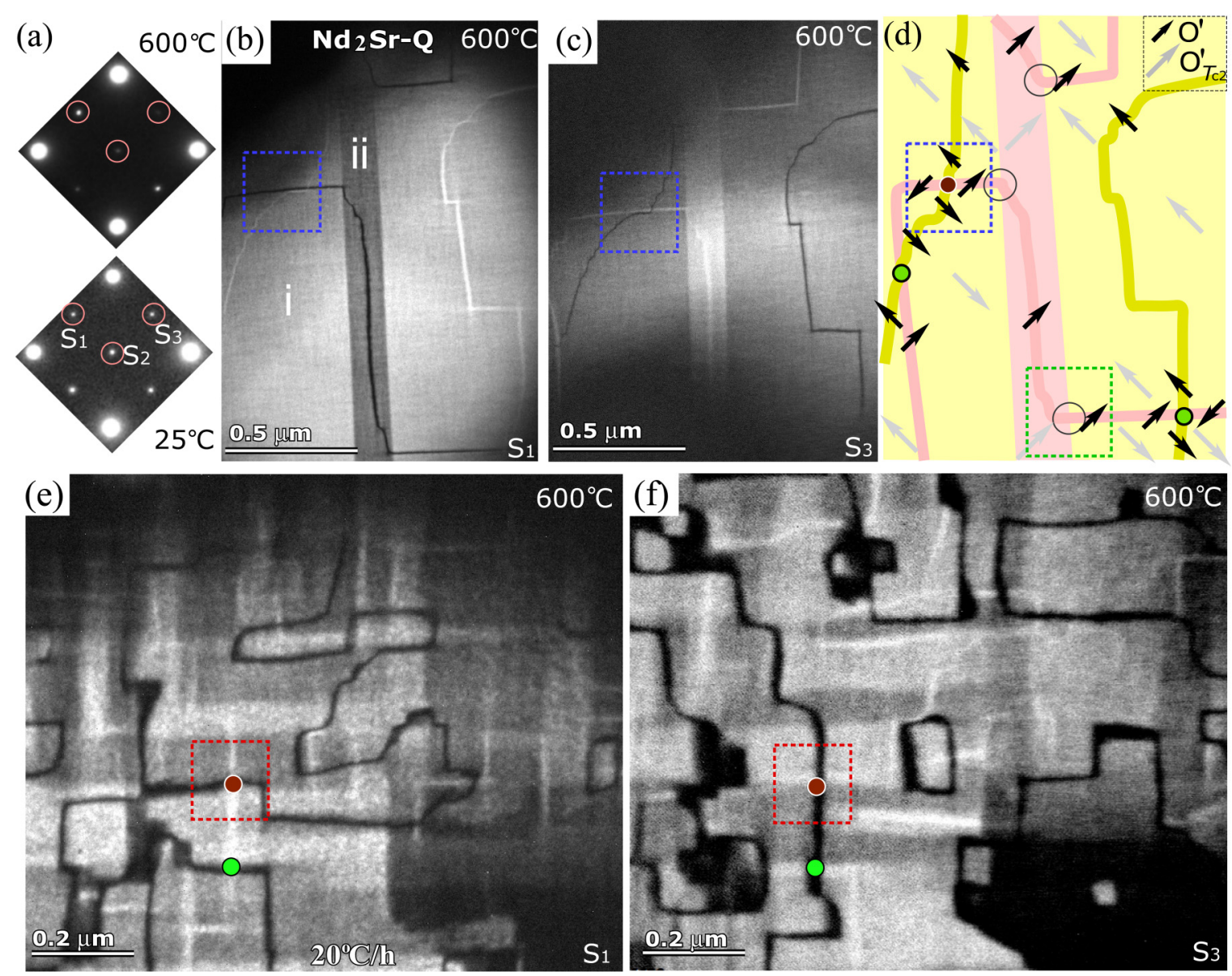

FIG. 5. In situ heating domain patterns in $\mathrm{Nd}_{2} \mathrm{SrFe}_{2} \mathrm{O}_{7}$ crystals. (a) $\mathrm{SAED}$ patterns at $25^{\circ} \mathrm{C}$ and $600^{\circ} \mathrm{C}$ (above $T_{\mathrm{c} 2}$ ), showing the $\mathrm{T}^{\prime}$ to $\mathrm{O}^{\prime}$ transition. Note that $\mathrm{S}_{2}=1 / 2(200)_{\mathrm{T}}$-type spots are not allowed in the $\mathrm{O}^{\prime}$ phase. The residual $\mathrm{S}_{2}$ intensity reflects the first-order nature of the transition. High-temperature DF-TEM images of (b), (c) $\mathrm{Nd}_{2} \mathrm{Sr}-\mathrm{Q}$ and (e), (f) $\mathrm{Nd}_{2} \mathrm{Sr}-20^{\circ} \mathrm{C} / \mathrm{h}$, taken using superlattice $\mathrm{S}_{1}$ and $\mathrm{S}_{3}$ spots. Four levels of color are identified: black, dark-gray, light-gray, and white. Black and white correspond to leftover $\mathrm{O}^{\prime}$-symmetry DWs, while dark(light)-gray contrasts relate to the nucleated $\mathrm{O}_{T \mathrm{c} 2}^{\prime}$ domains. The reversed contrast of those emerging rectangular dark- and light-gray $\mathrm{O}_{T \mathrm{c} 2}^{\prime}$ domains indicates $90^{\circ}$-crystallographic orthorhombic twin relation. (d) The corresponding vector map of (c), (d), showing $\mathrm{Z}_{4} \mathrm{O}_{T \mathrm{c} 2}^{\prime}$ (anti)vortices. Light-red and light-yellow blocks are $\mathrm{O}_{T \mathrm{c} 2}^{\prime}$ twin domains. Black and gray arrows represent the $\mathrm{O}^{\prime}$ phase with different tilt magnitudes. Brown and green circles depict immobilized vortex and antivortex cores. Partial (anti)vortices defined by an uncompleted cycle of vectors (order parameters) are circled. The domain evolutions in red and green dotted rectangles are shown in Figs. 6(b) and 6(c).

domain contrast of the nucleated $\mathrm{O}_{T \mathrm{c} 2}^{\prime}$ phase confirm our assumption.

Figure 6(b) illustrates two examples of $\mathrm{Z}_{4} \mathrm{O}^{\prime}{ }_{\mathrm{Tc} 2}$ vortex domains observed in Figs. 5(e) and 5(f). An appealing aspect is that those $\mathrm{T}^{\prime}$ vortex cores remain immobilized and become the $\mathrm{Z}_{4} \mathrm{O}_{T \mathrm{c} 2}^{\prime}$ vortex cores [Fig. 6(b)] upon heating. Note that the vortex cores may stay either at the twin boundaries [upper figures of Fig. 6(b)] or within a twin domain [lower figures of Fig. 6(b)]. Distinct from our proposed type II $\mathrm{Z}_{8} \mathrm{O}^{\prime}$ vortex or lamellar $\mathrm{O}^{\prime}$ twins and [left or right paths in Fig. 1(b)], surprisingly, $\mathrm{Z}_{4} \mathrm{O}_{T \mathrm{c} 2}^{\prime}$ (anti)vortices develop with the leftover $\mathrm{O}^{\prime}$-symmetry DWs captured within the nucleated $\mathrm{O}_{T \mathrm{c} 2}^{\prime}$ twins. In addition, partial (anti)vortices defined by an uncompleted cycle of vectors (order parameters) may form at the intersection of a nucleated $\mathrm{O}^{\prime}{ }_{T \mathrm{c} 2}$ twin boundary and a $\mathrm{O}^{\prime}$ wall as clearly depicted in Figs. 5(d) and 6(c).

The transition from $\mathrm{Z}_{8} \mathrm{~T}^{\prime}$ vortices to $\mathrm{Z}_{4} \mathrm{O}_{T \mathrm{c} 2}^{\prime}$ vortices is further explained by the evolution of energy landscapes across $T_{\mathrm{c} 2}$. At room temperature, the $\mathrm{T}^{\prime}$ phase is stable and the $\mathrm{O}^{\prime}$ phase is metastable as shown in Fig. 6(a). To reduce the interfacial energy, the stable vortex core structure is $Z_{8}$ $\mathrm{T}^{\prime}$ vortices with the $\mathrm{O}^{\prime}$ phase within the DWs. On the other hand, when the temperature is higher than $T_{c 2}$, the $\mathrm{T}^{\prime}$ phase becomes the $\mathrm{O}_{T \mathrm{c} 2}^{\prime}$ phase, while the $\mathrm{O}^{\prime}$ phase almost remains unchanged in the energy surfaces in Fig. 6(a). Due to the large energy barriers between the $\mathrm{O}_{\mathrm{Tc} 2}^{\prime}$ and $\mathrm{O}^{\prime}$ phase, the $\mathrm{O}^{\prime}$ phase maintains within the DWs as shown experimentally (Fig. 5). In contrast to the $\mathrm{T}^{\prime}$ phase, the $\mathrm{O}_{T \mathrm{c} 2}^{\prime}$ phase displays spontaneous distortion, and thus the domain structure shows straight twin boundaries. Note that the $\mathrm{O}_{T \mathrm{c} 2}^{\prime}$ and $\mathrm{O}^{\prime}$ phases are isosymmetric, and their difference is on the magnitude of structural distortions.

\section{SPONTANEOUS DISTORTION EFFECT ON DOMAIN PATTERNS}

Since type II $Z_{8} T^{\prime}$ topological defects are associated with octahedral distortions, we turn our attention to the effects 

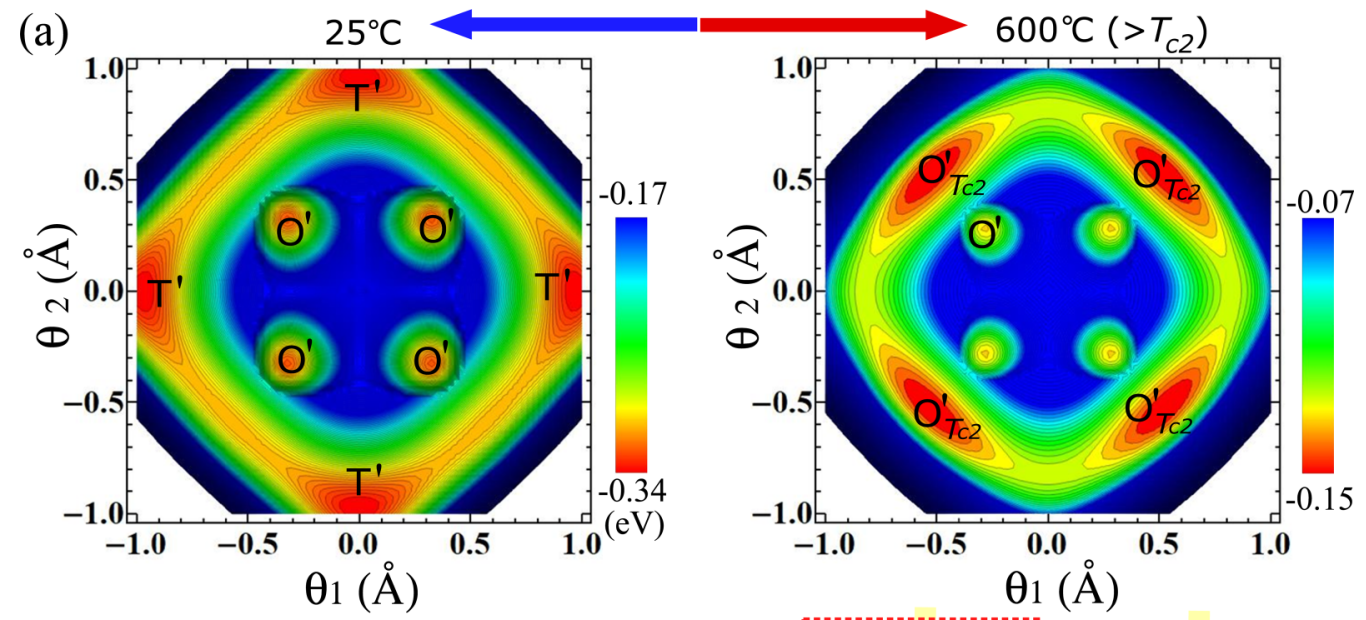

(b)
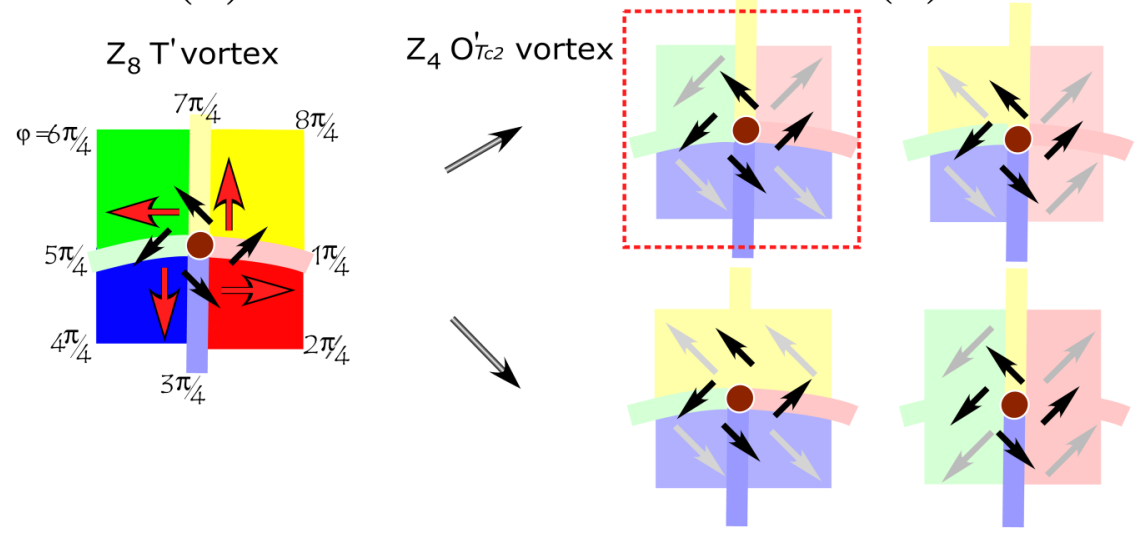

(c)
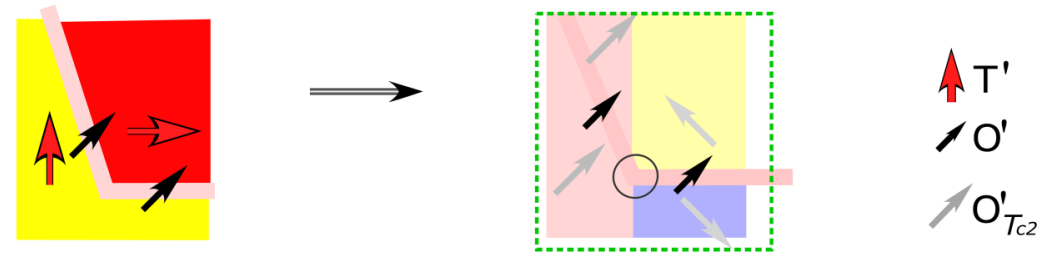

FIG. 6. Schematics for the energy contours and domain structure evolutions. (a) Energy landscapes with the temperature below and above $T_{\mathrm{c} 2}$. At $25^{\circ} \mathrm{C}$ (below $T_{\mathrm{c} 2}$ ) $\mathrm{T}^{\prime}$ corresponds to the stable state, while $\mathrm{O}^{\prime}$ is metastable. At $600^{\circ} \mathrm{C}$ (above $T_{\mathrm{c} 2}$ ), $\mathrm{O}^{\prime} T \mathrm{c} 2$ becomes stable, and $\mathrm{O}^{\prime}$ remains metastable. The energy landscapes are schematic plots for the energy surfaces at $25^{\circ} \mathrm{C}$ and $600^{\circ} \mathrm{C}$, which are suggested from our TEM images at those temperatures. (b) Schematic examples of $\mathrm{Z}_{4} \mathrm{O}^{\prime}{ }_{\mathrm{cc} 2}$ vortices and the evolutionary scheme. The color assignment is based on the order parameter direction as shown in Fig. 1(b). Red, black, and gray arrows represent $\mathrm{T}^{\prime}, \mathrm{O}^{\prime}$, and $\mathrm{O}_{\mathrm{Tc} 2}^{\prime}$ phases. (c) A partial vortex arises at the intersection of $\mathrm{O}^{\prime}{ }_{\mathrm{Tc} 2}$ twin boundary and one leftover $\mathrm{O}^{\prime}$-symmetry DWs (nanodomains).

of spontaneous distortion on the vortex formation. It is well known that the amplitude of local oxygen octahedral distortions in RP compounds can be manipulated by varying the ionic size mismatch (i.e., the so-called tolerance factor [37]). We have prepared high-quality polycrystalline samples of the solid solution $\mathrm{Nd}_{2} \mathrm{Ca}_{x} \mathrm{Sr}_{1-x} \mathrm{Fe}_{2} \mathrm{O}_{7}$ in the range of $0 \leqslant x \leqslant 0.6$, with the aim of increasing the tilt amplitude by the partial chemical substitutions of small $\mathrm{Ca}^{2+}$ for large $\mathrm{Sr}^{2+}$. We establish the phase diagram [Fig. 7(a)] and spontaneous distortion [Fig. 7(b)] of $\mathrm{Nd}_{2} \mathrm{Ca}_{x} \mathrm{Sr}_{1-x} \mathrm{Fe}_{2} \mathrm{O}_{7}$, as inferred from the in situ and ex situ domain observations during various heat treatments (see Appendix D). For example, we have observed orthorhombic twin-like structures at $x=0.5\left[\mathrm{Nd}_{2} \mathrm{Ca}_{0.5}-\mathrm{S}\right.$ in Fig. $\left.7(\mathrm{c})\right]$ at room temperature. Twins rearrange completely after the heat treatment up to $1470^{\circ} \mathrm{C}\left[\mathrm{Nd}_{2} \mathrm{Ca}_{0.5}-\mathrm{Q}\right.$ in Fig. $\left.7(\mathrm{~d})\right]$ while little changes with various thermal treatments below $1470^{\circ} \mathrm{C}$. Here capital Q and
$\mathrm{S}$ denote quenching and slow cooling processes, respectively, across both $T_{\mathrm{c} 1}$ and $T_{\mathrm{c} 2}$. Surprisingly, the electron-diffraction analysis reveals a persistent tetragonal $\mathrm{T}^{\prime}$ phase all the way to the composition $x=0.5$ at room temperature (see Appendix D). On the other hand, the existence of spontaneous distortion is evident, showing the peak broadening and splitting as a function of $\mathrm{Ca}$ content in $\mathrm{X}$-ray powder diffraction pattern [Fig. 7(b)]. The spontaneous distortion changes with different heat treatments [bottom panel of Fig. 7(a)]. The spontaneous distortion of $\Delta a / a=(b-a) / a$, where $a$ and $b$ are the orthorhombic lattice parameters, varies between 0 and $1.0 \%$ with the $\mathrm{Ca}$ substitution. $\mathrm{Nd}_{2} \mathrm{Ca}_{0.5}-\mathrm{S}$ has the largest $1 \%$ spontaneous distortion and the finest twin domains [Fig. 7(c)], while $\mathrm{Nd}_{2} \mathrm{SrFe}_{2} \mathrm{O}_{7}$ possesses zero distortion.

Figures 7(e) and 7(f) reveal microstructures of $\mathrm{Nd}_{2} \mathrm{Ca}_{0.5}-\mathrm{S}$ and $\mathrm{Nd}_{2} \mathrm{Ca}_{0.5}$-Q using superlattice DF-TEM images. A drastic change occurs in $\mathrm{Nd}_{2} \mathrm{Ca}_{0.5}-\mathrm{S}$ [Fig. 7(e)] wherein no type II 
(a) $\mathrm{T}(\mathrm{s}$

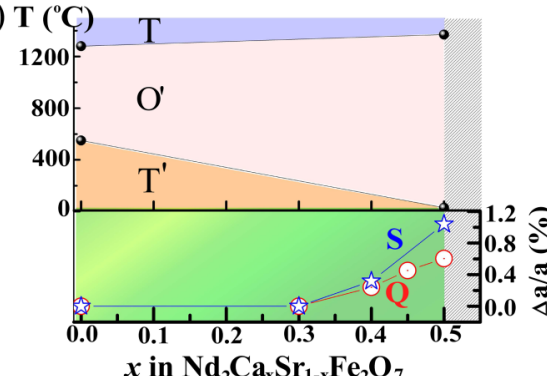

(b)

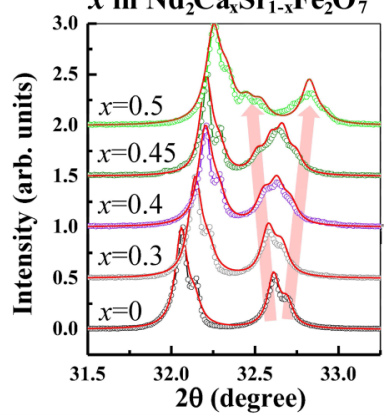

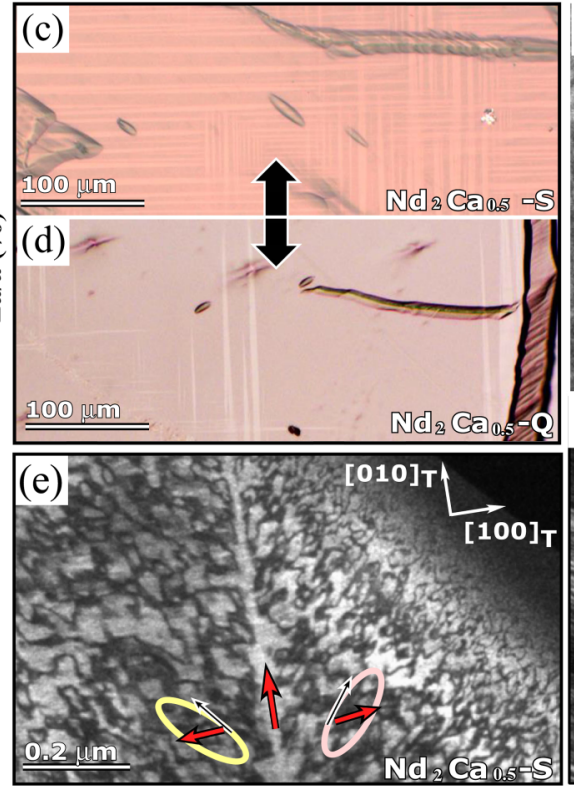

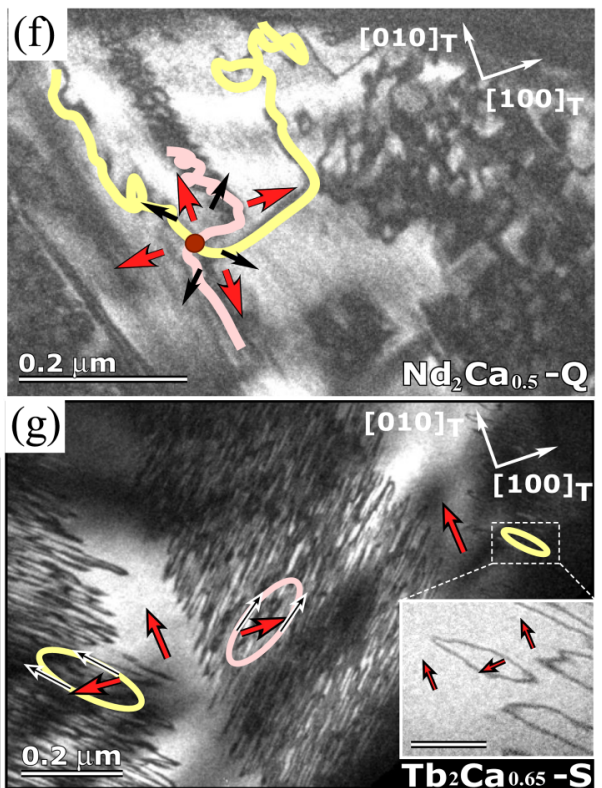

FIG. 7. (a) The phase diagram and the evolution of spontaneous distortion $(\Delta a / a)$ as a function of the $\mathrm{Ca}$ content in $\mathrm{Nd}_{2} \mathrm{Ca}_{x} \mathrm{Sr}_{1-x} \mathrm{Fe}_{2} \mathrm{O}_{7}$ with Q (red line) and S (blue line) heat treatments. The capital Q and S signify quenching and slow cooling with the rate of $100^{\circ} \mathrm{C} / \mathrm{h}$ from $1400-1470^{\circ} \mathrm{C}$ (above $T_{c 1}$ ). Spontaneous distortion $(\Delta a / a)$ changes with different heat treatments, which is closely related with the fine domain structure shown in panels (c) and (d). (b) X-ray data showing the peak broadening and splitting of the $(100)_{\mathrm{T}} /(010)_{\mathrm{T}}$ reflection as a function of $\mathrm{Ca}$ content. Raw and refined data are shown in color circles and red lines, respectively. Reflection-mode polarized optical microscope images of (c) $\mathrm{Nd}_{2} \mathrm{Ca}_{0.5}$-S with pseudotwins about tens of $\mu \mathrm{m}$ in size and (d) $\mathrm{Nd}_{2} \mathrm{Ca}_{0.5}-\mathrm{Q}$ with a low density of pseudotwins at room temperature. In-plane DF-TEM images of (e) $\mathrm{Nd}_{2} \mathrm{Ca}_{0.5}-\mathrm{S}$ and (f) $\mathrm{Nd}_{2} \mathrm{Ca}_{0.5}-\mathrm{Q}$, showing distinct microstructures at room temperature. The bright and dark contrasts are associated with $\mathrm{T}^{\prime}$ domain and $\mathrm{O}^{\prime} \mathrm{DWs}$, respectively. $\mathrm{Nd}_{2} \mathrm{Ca}_{0.5}-\mathrm{S}$ of $1 \%$ spontaneous distortion reveals periodic $\mathrm{O}^{\prime} \mathrm{DWs}$, while $\mathrm{Nd}_{2} \mathrm{Ca}_{0.5}-\mathrm{Q}$ of $0.6 \%$ spontaneous distortion shows local aggregation of high-density $\mathrm{O}^{\prime} \mathrm{DW}$. (g) The microscopic image of $\mathrm{Tb}_{2} \mathrm{Ca}_{0.65} \mathrm{Sr}_{0.35} \mathrm{Fe}_{2} \mathrm{O}_{7}$ of $0.7 \%$ spontaneous distortion, showing similar pseudotwins as $\mathrm{Nd}_{2} \mathrm{Ca}_{0.5}-\mathrm{S}$. Inset shows a blow-up image of $\mathrm{T}^{\prime} / \mathrm{O}^{\prime}$ loops with $90^{\circ}$ rotation of the neighboring $\mathrm{T}^{\prime}$ domains. Scale bar: $0.2 \mu \mathrm{m}$.

$\mathrm{Z}_{8} \mathrm{~T}^{\prime}$ vortex has been observed. The matrix with the bright contrast is still the $\mathrm{T}^{\prime}$ phase while DWs are dark. High-density dark wiggled loops self-group and elongate along either the $[110]_{\mathrm{T}}$ or $[\overline{1} 10]_{\mathrm{T}}$ diagonal direction. Those regions of elongated DWs alternate and form a quasiperiodic array over macroscopic distances and pseudotwins [see Fig. 7(e) and Appendix E]. The origin of these elongated closed loops becomes clear with a complementary domain-wall mapping (see Appendix E). Our DF-TEM images with different superlattice peaks indicate a similar orthorhombic-like local structure of those loops, i.e., $\mathrm{T}^{\prime} / \mathrm{O}^{\prime}$ loops, loop domain walls with $\mathrm{O}^{\prime}$ distortions in the $\mathrm{T}^{\prime}$ background/domains. In addition, the elongated direction is associated with two different tilt axes. Therefore, the orthorhombic twin-like structure observed under a polarized optical microscope [see Figs. 7(c) and 7(d) and Appendix E] is, in fact, associated with pseudo wins in the $\mathrm{T}^{\prime}$ matrix with alternating two types of $\mathrm{T}^{\prime} / \mathrm{O}^{\prime}$ loops: each type of orthorhombic loops conglomerate periodically. We emphasize that this is highly unusual, since the matrix is the $\mathrm{T}^{\prime}$ phase, and no twinning would be expected in a tetragonal structure in general.

A similar pattern was found in $\mathrm{Tb}_{2} \mathrm{Ca}_{0.65} \mathrm{Sr}_{0.35} \mathrm{Fe}_{2} \mathrm{O}_{7}$ [Fig. $7(\mathrm{~g})$ ], where those $\mathrm{T}^{\prime} / \mathrm{O}^{\prime}$ loops tend to sharpen up and avoid mutual intersections. As shown in the inset of Fig. $7(\mathrm{~g})$, inside and outside a loop, the tilt directions of two $\mathrm{T}^{\prime}$ domains always rotate $90^{\circ}$ (red arrows). Note that the background matrix dominant in terms of volume is the $\mathrm{T}^{\prime}$ phase, and one out of four $\mathrm{T}^{\prime}$ states is favored in an extended region (red arrows outside the loops). Interestingly, the partially restoration of type II $\mathrm{Z}_{8} \mathrm{~T}^{\prime}$ vortices occurs in $\mathrm{Nd}_{2} \mathrm{Ca}_{0.5}-\mathrm{Q}$ with a fast-quenching process across $T_{\mathrm{c} 1}$, resulting in a low density of pseudotwins [Figs. 7(d) and 7(f)]. The size of local patches varies in a wide range from tens of nanometers to submicron. Inside the submicron domains of one type of $\mathrm{T}^{\prime} / \mathrm{O}^{\prime}$ loops, there is still contained small patches of the other type of $\mathrm{T}^{\prime} / \mathrm{O}^{\prime}$ loops. A complete analysis can be found in Appendix E. Those local patches can be considered as nanotwins and should behave invisible under a polarized optical microscope, which explains the reduction of optically observable twin-like structure in Fig. 7(d).

We then discuss the underlying mechanisms behind the formation of vortices and $\mathrm{T}^{\prime} / \mathrm{O}^{\prime}$ loops based on phase-field simulations [Figs. 8(a) and 8(b)]. When the temperature is between $T_{\mathrm{c} 1}$ and $T_{\mathrm{c} 2}$, the stable domain structure is orthorhombic twins with the possible coexistence of $\mathrm{Z}_{4} \mathrm{O}_{T \mathrm{c} 2}^{\prime}$ vortices. For a slow cooling across $T_{\mathrm{c} 1}$ and large spontaneous distortion, the $\mathrm{Z}_{4} \mathrm{O}_{T \mathrm{c} 2}^{\prime}$ vortex density is very low. For simplicity, the initial domain configuration in our simulation is an $\mathrm{O}_{T \mathrm{c} 2}^{\prime}$ twin as shown in Fig. 8(a), with the two domains corresponding to $\varphi=1 \pi / 4$ (light red) and $7 \pi / 4$ (light yellow), respectively. When the temperature is cooled below $T_{\mathrm{c} 2}, \mathrm{~T}^{\prime}$ domains will replace $\mathrm{O}_{T \mathrm{c} 2}^{\prime}$ domains. According to the energy landscape shown in Fig. 1(c), the yellow $\mathrm{T}^{\prime}$ domain $(\varphi=8 \pi / 4)$ naturally becomes the majority matrix since the corresponding 


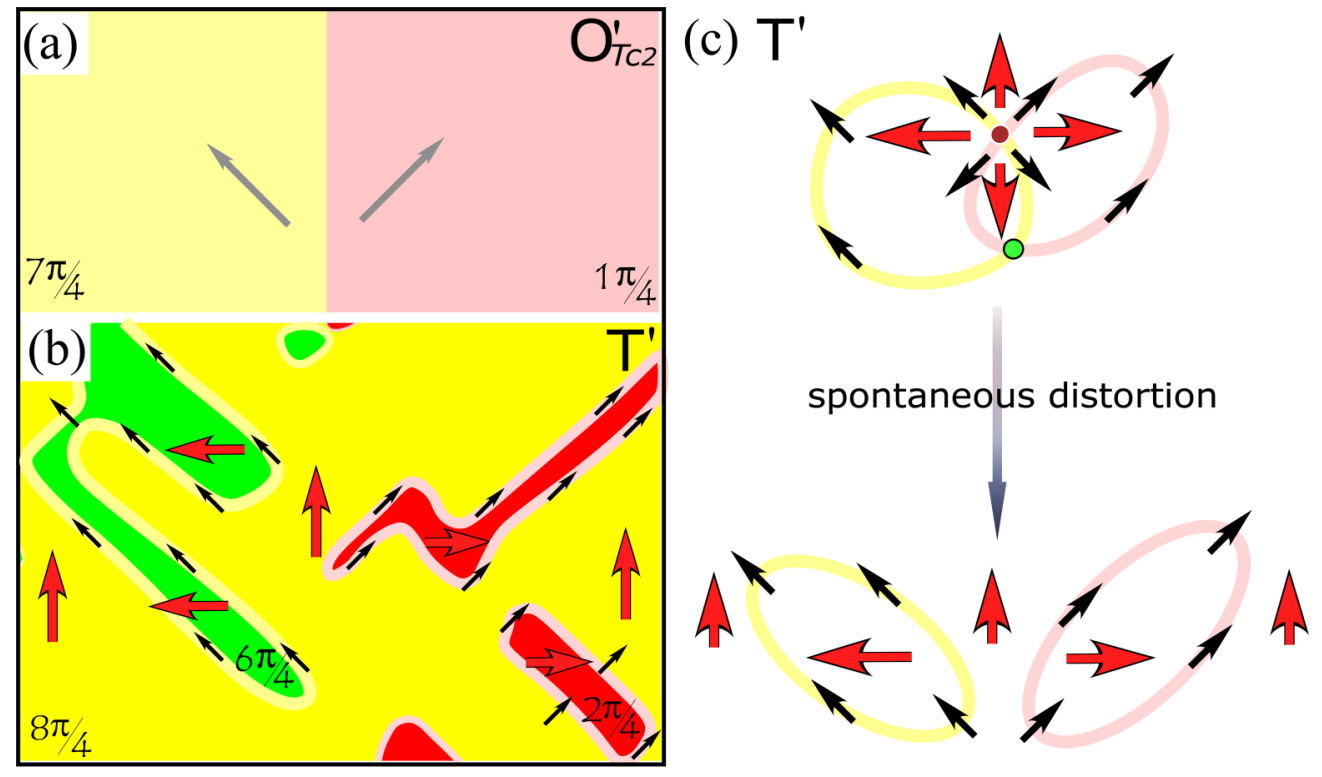

FIG. 8. (a), (b) Phase-filed simulation for the domain structure evolution across the $T_{c 2}$ transition, starting from (a) initial high-temperature $\mathrm{O}_{T \mathrm{c} 2}^{\prime}$ twin domains to (b) low-temperature $\mathrm{T}^{\prime} / \mathrm{O}^{\prime}$ loops with $\varphi=8 \pi / 4$ (yellow) as the major $\mathrm{T}^{\prime}$ phase. Order parameter direction is denoted by arrows and colors as shown in Fig. 1(b). (c) Schematics of the formation of type II $Z_{8} \mathrm{~T}^{\prime}$ vortices and $\mathrm{T}^{\prime} / \mathrm{O}^{\prime}$ loops in the presence of spontaneous distortion at room temperature.

order parameter is close to the two initial $\mathrm{O}_{T \mathrm{c} 2}^{\prime}$ domains. The red $(\varphi=2 \pi / 4)$ and green $(\varphi=6 \pi / 4) \mathrm{T}^{\prime}$ domains grow as inclusions within the matrix, and the boundaries of the inclusion are $\mathrm{O}^{\prime}$ nanodomains [Fig. 8(b)], consistent with the experimental observation [see Fig. 7(e) and Appendix E]. The full evolution process is demonstrated in a supplemental movie [38]. The elimination of the blue $\mathrm{T}^{\prime}$ domain $(\varphi=4 \pi / 4)$ can be understood as a long switching path or a high-energy barrier across the center peak [Fig. 1(c)]. Evidently, the enhanced spontaneous distortion by chemical substitutions of $\mathrm{Ca}^{2+}$ for $\mathrm{Sr}^{2+}$ favors well-defined large-scale lamellar $\mathrm{O}^{\prime}$-orthorhombic twins at the intermediate state and results in the transformation from type $\mathrm{II} \mathrm{Z}_{8} \mathrm{~T}^{\prime}$ vortex to $\mathrm{T}^{\prime} / \mathrm{O}^{\prime}$ loops [Fig. 8(c)]. Note that, in $\mathrm{Na}_{2} \mathrm{Ca}_{0.4}$ within a moderate spontaneous distortion, one can completely manipulate the $\mathrm{T}^{\prime} / \mathrm{O}^{\prime}$ loops or the type II $\mathrm{Z}_{8} \mathrm{~T}^{\prime}$ vortices state with different heat treatments (see Appendix E).

\section{CONCLUSIONS}

In summary, we have discovered and explored the formation of topological defects of type II $\mathrm{Z}_{8} \mathrm{~T}^{\prime}$ (anti)vortices at room temperature, $\mathrm{Z}_{4} \quad \mathrm{O}_{T \mathrm{c} 2}^{\prime}$ (anti)vortices at high temperatures, and $\mathrm{T}^{\prime} / \mathrm{O}^{\prime}$ loops in the presence of significant spontaneous distortion, traversing successive second-order and first-order phase transitions in a bilayered ferrite. We demonstrate that the dynamics at the second-order critical temperature plays the decisive role in determining the final density of topological type II $\mathrm{Z}_{8}$ (anti)vortices with a power-law dependence, following the Kibble-Zurek scaling mechanism. Upon heating across $T_{\mathrm{c} 2}$, the vortex cores are not affected by symmetry breaking and become the cores of $\mathrm{Z}_{4} \mathrm{O}_{T \mathrm{c} 2}^{\prime}$ (anti)vortices. The coexistence of $\mathrm{O}^{\prime}$ phases with different distortion amplitudes during in situ heating implies a locally preserved structure and hysteretic and complex fluc- tuations/variations of the $X_{3}^{-}$mode order-parameter angles in the first-order type $T_{\mathrm{c} 2}$ transition. The expected vortex-tovortex transformation outlined in the left path of Fig. 1(b) was not observed in our system but may require a system with two continuous (i.e., second-order) phase transitions. In addition, unprecedented type II $\mathrm{Z}_{8} \mathrm{~T}^{\prime}$ (anti)vortex to $\mathrm{T}^{\prime} / \mathrm{O}^{\prime}$ loop evolution, occurring with enhanced spontaneous distortion, represents a finely balanced set of tilt order parameters, which may enable external thermodynamic and strain/pressure control. It should be further investigated how these real-space crystallographic order parameter topologies presented here influence the configurations of antiferromagnetic domains and DWs through spin-lattice coupling in this bilayer ferrite. Furthermore, there are numerous materials undergoing multiple structural and/or magnetic phase transitions, and our findings are a stepping stone to study the evolution of topological defects in any of those materials.

\section{ACKNOWLEDGMENTS}

The work at Rutgers was funded by the DOE under Grant No. DE-FG02-07ER46382 to the Rutgers Center for Emergent Materials. The work at Penn State was supported as part of the Computational Materials Sciences Program funded by the U.S. Department of Energy, Office of Science, Basic Energy Sciences, under Award No. DE-SC0020145.

\section{APPENDIX A: PHASE-FIELD SIMULATIONS}

To describe the structural distortion in the system, we introduce a two-dimensional order parameter $\left(\theta_{1}, \theta_{2}\right)$, which represents the oxygen octahedral tilt along the pseudotetragonal axes. The bulk energy of the system is expressed by a Landau polynomial. Since the $\mathrm{T}^{\prime}$ phase is stable while the $\mathrm{O}^{\prime}$ phase is metastable, the bulk energy is expanded to the sixth 


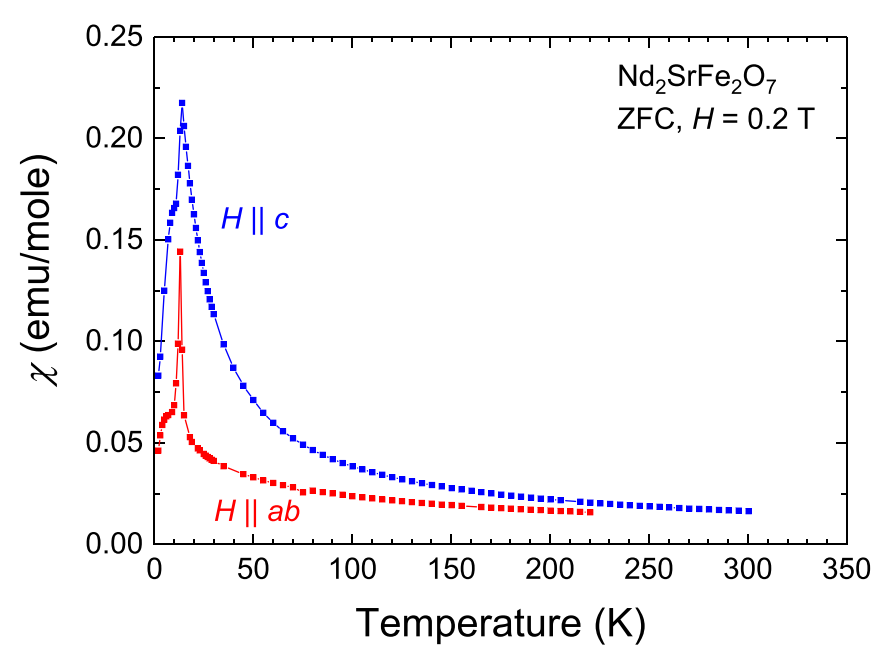

FIG. 9. Magnetic susceptibility curves of single crystals of $\mathrm{Nd}_{2} \mathrm{SrFe}_{2} \mathrm{O}_{7}$ measured under magnetic field of $0.2 \mathrm{~T}$ after zero field cooling (ZFC).

order [32]:

$$
\begin{aligned}
f_{\text {bulk }}= & b_{1}\left(\theta_{1}^{2}+\theta_{2}^{2}\right)+b_{11}\left(\theta_{1}^{4}+\theta_{2}^{4}\right)+b_{12} \theta_{1}^{2} \theta_{2}^{2} \\
& +b_{111}\left(\theta_{1}^{6}+\theta_{2}^{6}\right)+b_{112}\left(\theta_{1}^{4} \theta_{2}^{2}+\theta_{1}^{2} \theta_{2}^{4}\right) .
\end{aligned}
$$

In Eq. (A1), $b_{1}, b_{11}, b_{12}, b_{111}$, and $b_{112}$ are coefficients of the Landau polynomial under stress-free conditions, which
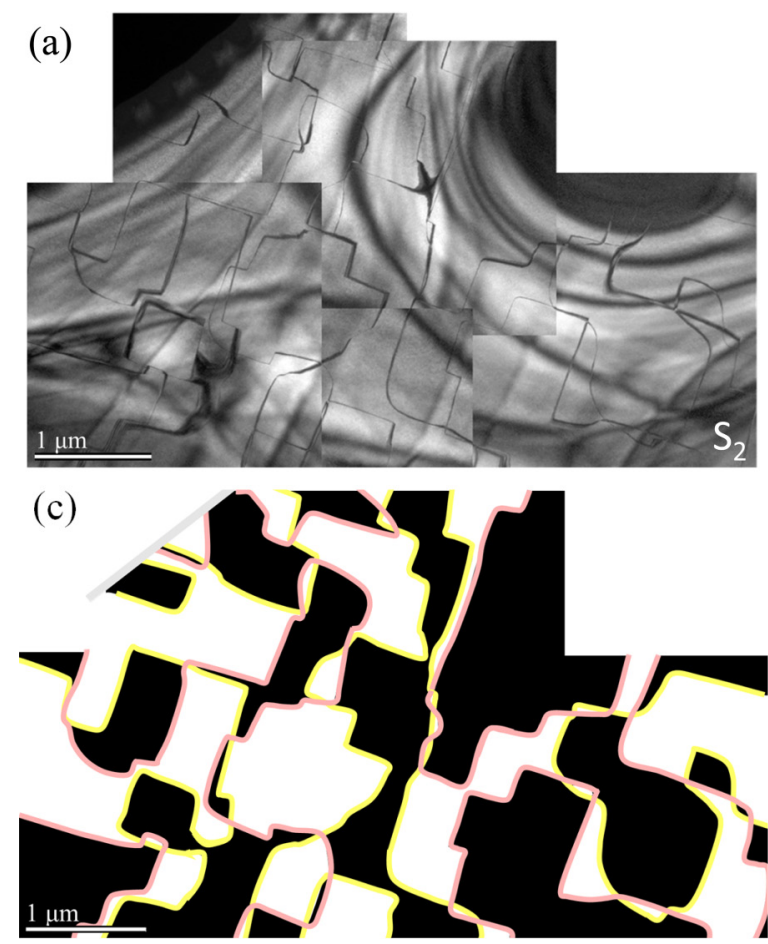

TABLE I. Coefficients of $\mathrm{Nd}_{2} \mathrm{SrFe}_{2} \mathrm{O}_{7}$ used in the phase-field simulations.

\begin{tabular}{lccc}
\hline \hline$b_{1}$ & $-0.1388 \mathrm{eV} \mathrm{\AA}^{-2}$ & $\kappa_{1212}$ & $8.80 \mathrm{eV}$ \\
$b_{11}$ & $-0.0010 \mathrm{eV} \mathrm{\AA}^{-4}$ & $c_{1111}$ & $281.14 \mathrm{GPa}$ \\
$b_{12}$ & $-0.3083 \mathrm{eV}^{-4}$ & $c_{1122}$ & $69.21 \mathrm{GPa}$ \\
$b_{111}$ & $0.0034 \mathrm{eV}^{-6}$ & $c_{1212}$ & $72.78 \mathrm{GPa}$ \\
$b_{112}$ & $0.1405 \mathrm{eV}^{-6}$ & $h_{1111}$ & $0.002064 \AA^{-2}$ \\
$\kappa_{1111}$ & $8.80 \mathrm{eV}$ & $h_{1122}$ & $-0.007513 \AA^{-2}$ \\
$\kappa_{1122}$ & $-8.80 \mathrm{eV}$ & $h_{1212}$ & $-0.04783 \AA^{-2}$ \\
\hline \hline
\end{tabular}

are fitted based on the corresponding coefficients of $\mathrm{Ca}_{3} \mathrm{Ti}_{2} \mathrm{O}_{7}$ [33]. Note that the magnitude of the order parameter does not represent the displacement of one oxygen atom of one octahedron. Instead, it is one type of summation of the displacement of all the oxygen atoms that involve in the $X_{3}^{-}$mode tilt in a unit cell, and the magnitude of the order parameter is extracted using the online software AMPLIMODES [34-36,39]. The Cartesian coordinates $\left(\theta_{1}, \theta_{2}\right)$ can be transformed to the polar coordinates $(Q, \varphi)$ through $\theta_{1}=Q \cos \varphi, \theta_{2}=Q \sin \varphi$. Here $Q$ and $\varphi$ are the magnitude and phase of $X_{3}^{-}$tilt mode, respectively. Then Eq. (A1) can be rewritten as

$$
\begin{aligned}
f_{\text {bulk }}= & b_{1} Q^{2}+\frac{1}{8}\left[\left(6 b_{11}+b_{12}\right) Q^{4}+\left(2 b_{11}-b_{12}\right) Q^{4} \cos 4 \varphi\right. \\
& \left.+\left(5 b_{111}+b_{112}\right) Q^{6}+\left(3 b_{111}-b_{112}\right) Q^{6} \cos 4 \varphi\right] .
\end{aligned}
$$
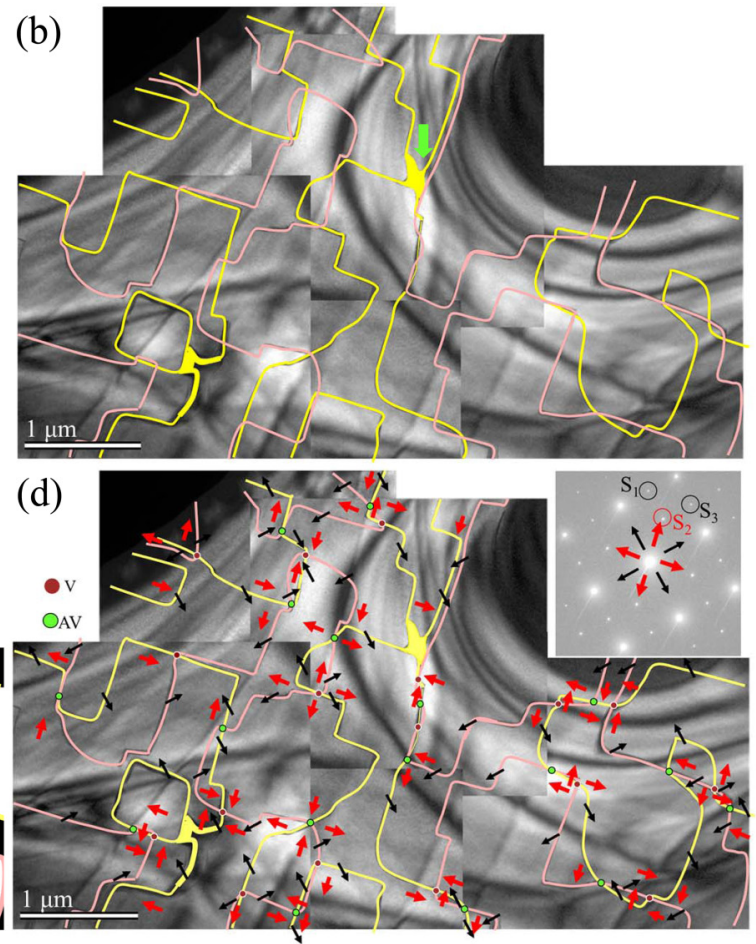

FIG. 10. (a) A $6 \times 4 \mu \mathrm{m}^{2}$ mosaic of DF-TEM images were taken on the $\mathrm{Nd}_{2} \mathrm{SrFe}_{2} \mathrm{O}_{7}$ crystal with $100^{\circ} \mathrm{C} / \mathrm{h}$ cooling rate. A cut-through graph can be readily constructed as shown in (b) based on the method described in Fig. 3. Light-yellow lines never cross any light-yellow lines, and light-red lines never cross any light-red lines. The likely crossing of light-yellow lines indicated by the green arrow is due to the projection nature in the TEM technique. (c) Two-proper coloring, black and white, is sufficient to identify the domains without the neighboring domains sharing the same color. The black-and-white $\mathrm{T}^{\prime}$ domains are related with $[100]_{\mathrm{T}}$ and $[010]_{\mathrm{T}}$ tilt axes. (d) The full mapping of eight variants in the domains and domain walls and the full identification of vortices (brown) and antivortices (green). A vortex is always surrounded by antivortices connected with DWs and vice versa. 
(a)

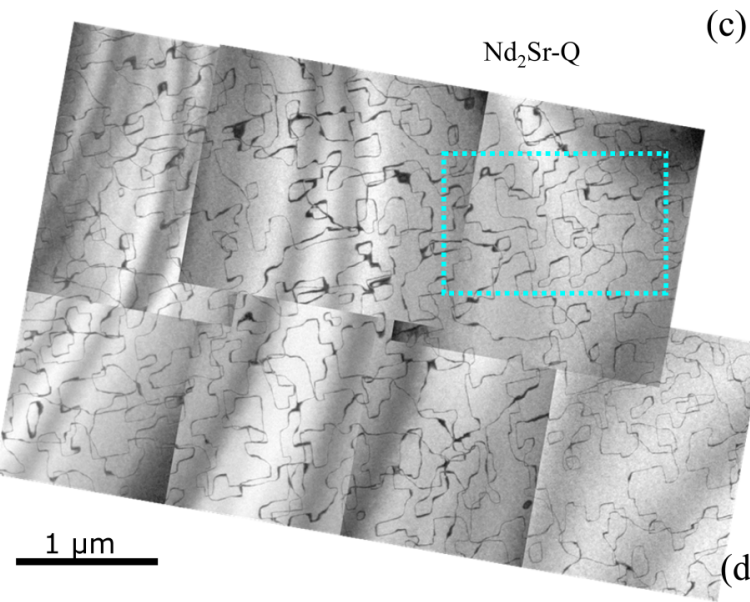

(b)

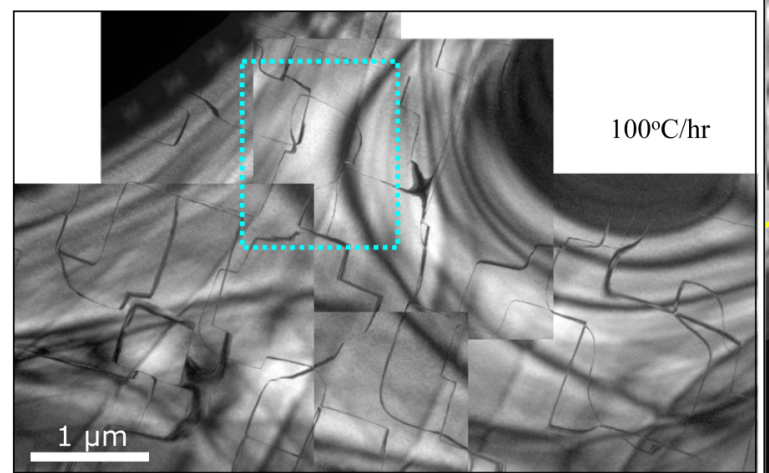

(c)

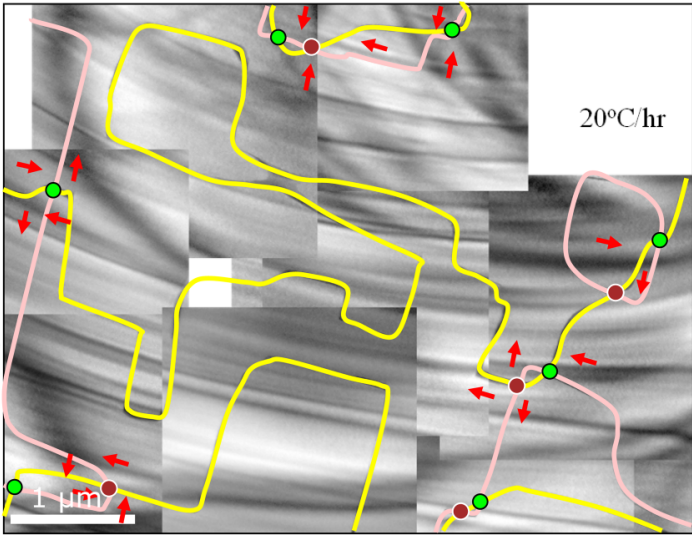

d)

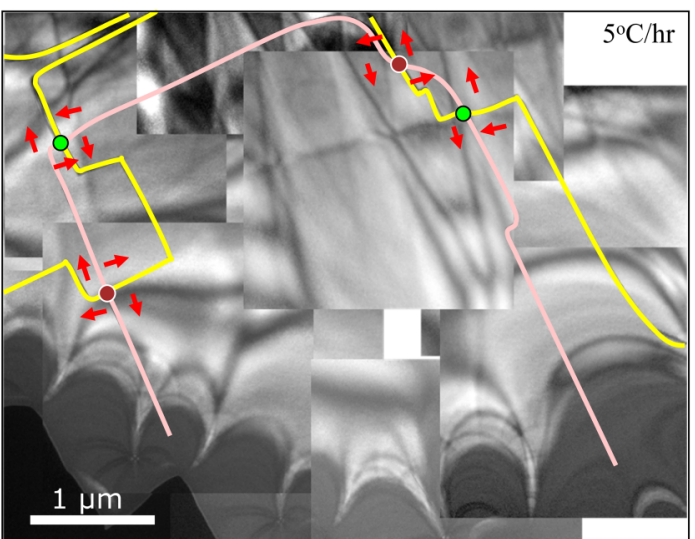

FIG. 11. Spatial maps of the type II $\mathrm{Z}_{8} \mathrm{~T}^{\prime}$ (anti)vortices in $\mathrm{Nd}_{2} \mathrm{SrFe}_{2} \mathrm{O}_{7}$ for a range of different cooling rates through $T_{\mathrm{c} 1}$. DF-TEM images were recorded with the $\mathrm{S}_{2}$ spot, covering $\sim 23 \mu \mathrm{m}^{2}$ for each panel on which vortex-antivortex pair analysis has been performed. The defect density D (core number/area): (a) $21.6 \mu \mathrm{m}^{-2}$, (b) $1.85 \mu \mathrm{m}^{-2}$, (c) $0.59 \mu \mathrm{m}^{-2}$, and (d) $0.17 \mu \mathrm{m}^{-2}$. The cooling-rate dependence of the vortex density follows a power law $\left\{D \propto t^{n}\right\}$ with the exponent $n$ of 0.59 as shown in the inset of Fig. 4(d). Four datapoints were covered in the fitting. Cyan rectangles mark the corresponding images shown in Fig. 4(a). by

In the phase-field simulations, the total free energy is given

$F=\int\left[f_{\text {bulk }}+\kappa_{i j k l} \frac{\partial \phi_{i}}{\partial x_{j}} \frac{\partial \phi_{k}}{\partial x_{l}}+\frac{1}{2} c_{i j k l}\left(\varepsilon_{i j}-\varepsilon_{i j}^{0}\right)\left(\varepsilon_{k l}-\varepsilon_{k l}^{0}\right)\right] d V$,

where $\kappa_{i j k l}$ are gradient energy coefficients, $c_{i j k l}$ is the elastic stiffness tensor, and $\varepsilon_{i j}$ and $\varepsilon_{k l}^{0}$ are the total strain and eigenstrain. The eigenstrain is related to the order parameter through $\varepsilon_{i j}^{0}=h_{i j k l} \theta_{k} \theta_{l}$, where $h_{i j k l}$ are coupling coefficients. The time-dependent Ginzburg-Landau (TDGL) equations are solved based on a semi-implicit spectral method [34]. Periodic boundary conditions are employed along the three directions. The system size is $1024 \Delta x \times 1024 \Delta x \times 1 \Delta x$, and the grid spacing is $\Delta x=0.20 \mathrm{~nm}$. The coefficients used in the simulation are listed in Table I.

\section{APPENDIX B: MAGNETIC MEASUREMENTS}

Magnetic measurements were carried out on single crystals using a commercial superconducting quantum interference device (SQUID) magnetometer (Quantum Design, USA). Figure 9 reveals rare-earth ordering at temperature below 15 $\mathrm{K}$ with spins aligned along the $c$-axis while it is antiferromagnetic (AFM) with spins in the plane at $300 \mathrm{~K}$. The AFM ordering takes place at a temperature 500-600 K [27-29].
Magnetism in (layered) perovskites tends to be strongly coupled with structural distortions, so this unique domain topology may not be limited to structural domains and DWs.

\section{APPENDIX C: MICROSTRUCTURES OF $\mathrm{Nd}_{2} \mathrm{SrFe}_{2} \mathrm{O}_{7}$}

In order to reveal domain patterns, thin-plate-like crystals were prepared by mechanical polishing, followed by Ar-ion milling, and studied using a JEOL-2010F TEM. We observed bright-contrast domains and DWs as dark lines with superlattice DF-TEM imaging taking $\mathrm{S}_{2}=1 / 2(200)_{\mathrm{T}}=(110)_{\mathrm{T}^{\prime}}$-type Bragg spots (Figs. 10 and 11). All images are raw data. Figure 10(b) maps out $\mathrm{O}^{\prime}$-symmetry DWs with light-yellow
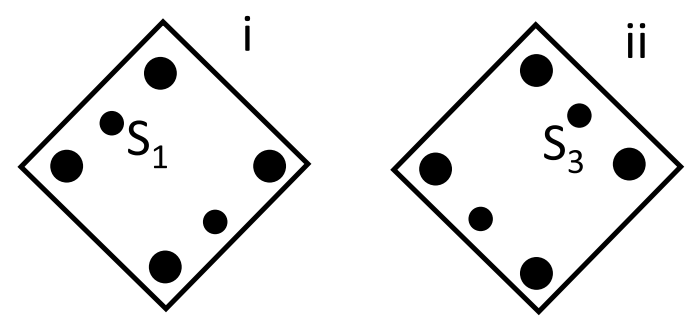

FIG. 12. (a) Schematics of diffraction patterns of regions (i) and (ii) in Fig. 5(b), showing the $90^{\circ}$ crystallographic-twin relation of regions (i) and (ii). 


\section{(a)}

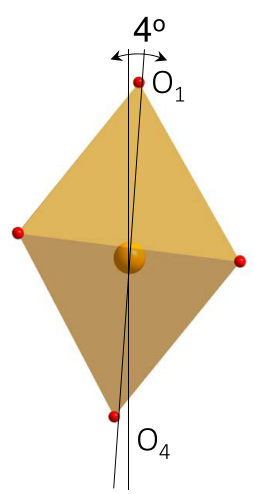

(b)

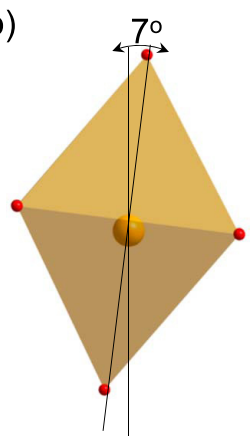

(c)

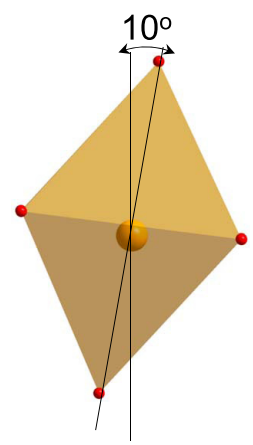

\begin{tabular}{|l|l|l|l|}
\hline$\theta_{\text {Tilt }}$ & $4^{\circ}$ & $7^{\circ}$ & $10^{\circ}$ \\
\hline$O_{1}(x y z)$ & $0.75,0.2836,0$ & $0.75,0.3036,0$ & $0.75,0.3236,0$ \\
\hline$O_{4}(x y z)$ & $0.25,0.2727,0.29522$ & $0.25,0.2927,0.29522$ & $0.25,0.3127,0.29522$ \\
\hline$F(120)^{*}$ & 63.18 & 57.7 & 52.8 \\
\hline
\end{tabular}

FIG. 13. Schematics of $\mathrm{FeO}_{6}$ octahedra with different tilting amplitude. $\mathrm{O}_{1}$ and $\mathrm{O}_{4}$ determine $\theta_{\text {tilt }}$ angle of (a) $4^{\circ}$, (b) $7^{\circ}$, and (c) $10^{\circ}$. Panel (a) represents the reported $\mathrm{O}^{\prime}$ structure adopted from Ref. [26], while (b) and (c) are artificially tuned $\mathrm{O}_{1}$ and $\mathrm{O}_{4}$ positions. The $\mathrm{F}_{(120)}$ structural factor is calculated on the assumption that those heavy $\mathrm{Sr} / \mathrm{Nd} / \mathrm{Fe}$ elements remain for different tilting models in the formula $|F(120)|^{2}=$ $[-16 \sin (2 \pi x) \sin (4 \pi y)]^{2}$ according to the $\mathrm{O}^{\prime}$ Amam (SG: 63) setting in the International Table for Crystallography volume B [40]. The calculated structural factors using the software Diamond [41] are shown. A larger tilting angle leads to a smaller $\mathrm{F}_{(120)}$ and a weaker contrast under DF-TEM.

and light-red colors based on the method described in Fig. 3. Two-proper coloring is also applied to $\mathrm{T}^{\prime}$ domain mapping as shown in Fig. 10(c) in such a way that any two bordering domains never have the same color-this is called proper coloring. Considering a pure tilting nature of the matrix $\mathrm{T}^{\prime}$ state and the distinct extinction rules; those DWs are likely in the $\mathrm{O}^{\prime}$ symmetry. The directions of oxygen octahedral tilts are denoted as red and black arrows [Fig. 10(d)]. Therefore, oxygen octahedra inside the bright contrast domains tilt along either the $[100]_{\mathrm{T}}$ or $[010]_{\mathrm{T}}$ directions (red arrows), whereas those in the dark-contrast DWs tilt along diagonal $\langle 110\rangle_{\mathrm{T}}$ directions (black arrows). Figure 11 shows superlattice DFTEM imaging taking $S_{2}=1 / 2(200)_{\mathrm{T}}=(110)_{\mathrm{T}^{\prime}}$ for a range of different cooling rates through $T_{\mathrm{c} 1}$. The specimens with 5 to $100^{\circ} \mathrm{C} / \mathrm{h}$ cooling rates across $T_{\mathrm{c} 1}$ were precisely controlled by the furnace setting. The quench experiments were performed with a small specimen staying above $T_{\mathrm{c} 1}$ for a few tens of minutes and then with the furnace door open till the furnace thermocouple reading drop across $T_{\mathrm{c} 1}$ for a $100^{\circ} \mathrm{C}$ interval. The quenching rate is estimated by taking a video of the quenching process to obtain the time consumption. The largest vortex density of about $21.6 \mu \mathrm{m}^{-2}$ is experimentally observed at the highest cooling rate $7000^{\circ} \mathrm{C} / \mathrm{h}$ as shown in Fig. 11(a). We note that the fitting adjustments may be needed due to statistical errors from the quench rate estimation and the limited observable areas utilizing the DF-TEM technique; however, the scaling of vortex density with various cooling rates across $T_{\mathrm{c} 1}$ instead of $T_{\mathrm{c} 2}$ is evident. (a)

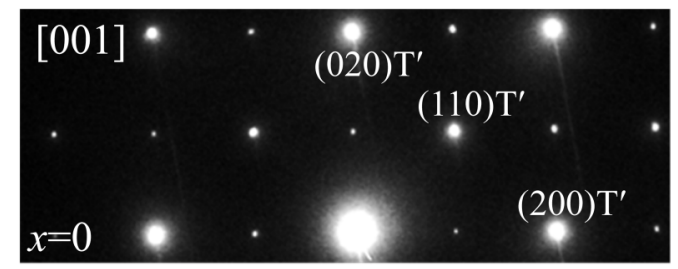

(b)

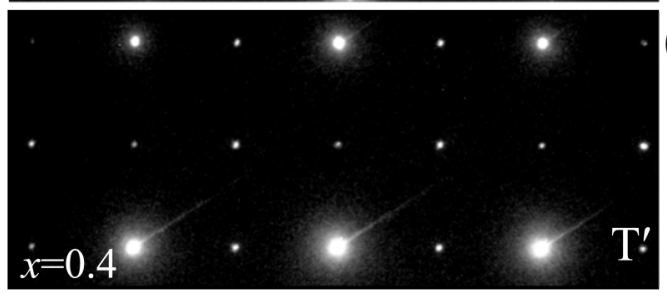

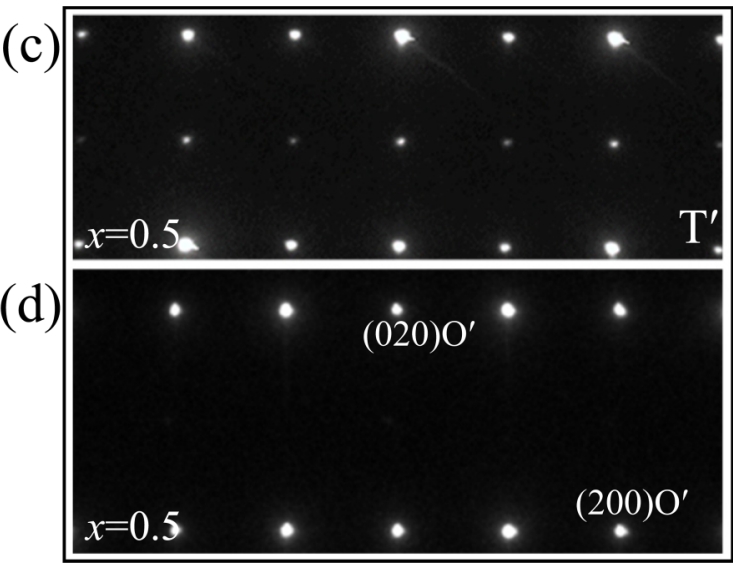

FIG. 14. Selected-area electron diffraction (SAED) patterns of $\mathrm{Nd}_{2} \mathrm{Ca}_{x} \mathrm{Sr}_{1-x} \mathrm{Fe}_{2} \mathrm{O}_{7}$ single crystals showing $\mathrm{T}^{\prime}\left(P 4_{2} / m n m\right)$ symmetry along [001] projection. (a) $x=0$, (b) $x=0.4$, and (c) $x=0.5$. The SAED pattern showing $\mathrm{O}^{\prime}$ (Amam) symmetry was found in (d) $x=0.5$ poly crystallines as a minority phase, which indicates the $\mathrm{T}^{\prime}-\mathrm{O}^{\prime}$ phase boundary close to $x=0.5$. 


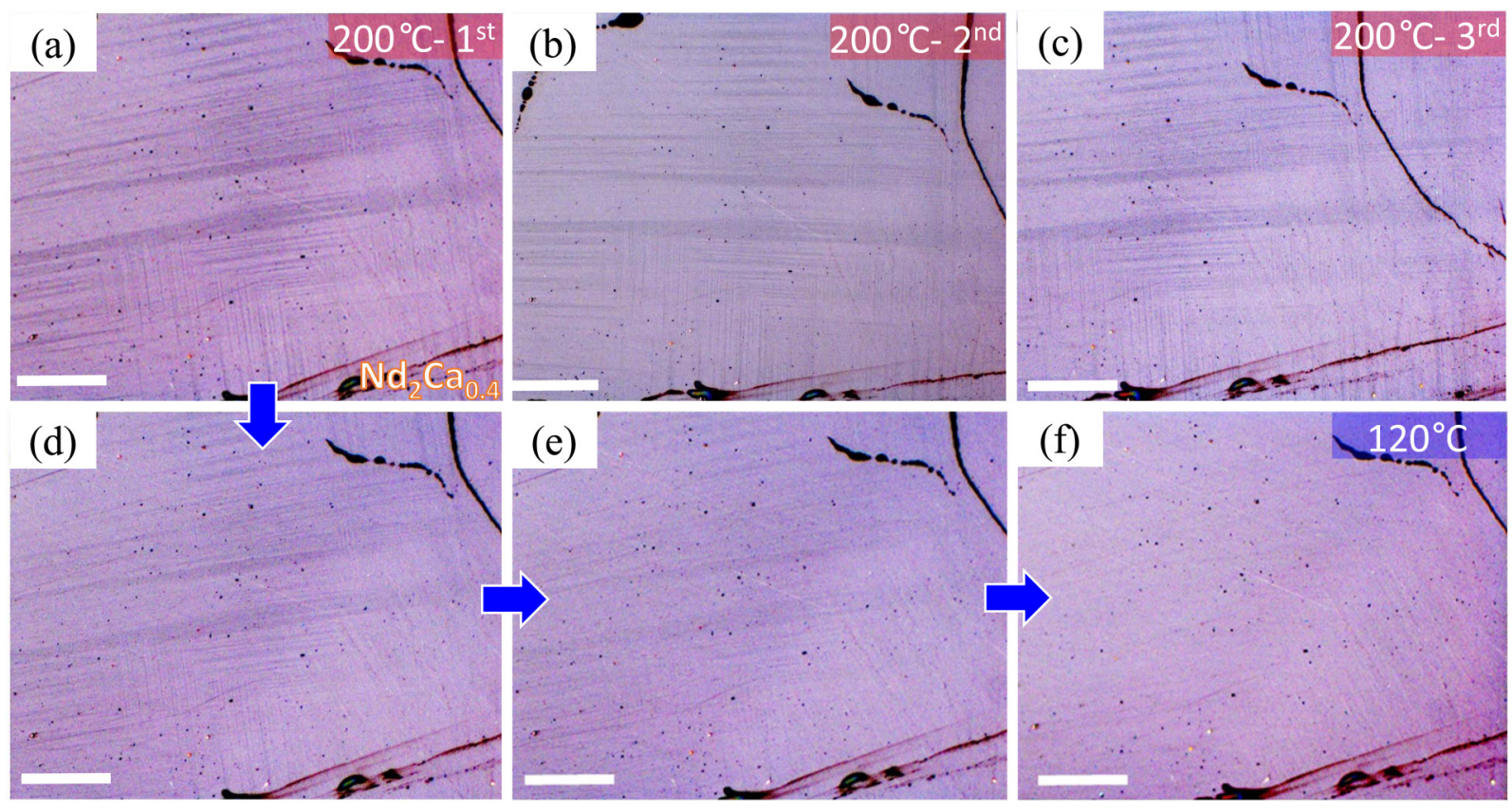

FIG. 15. Reflection mode polarized microscope images of real-time dynamics of $\mathrm{Nd}_{2} \mathrm{Ca}_{0.4} \mathrm{Sr}_{0.6} \mathrm{Fe}_{2} \mathrm{O}_{7}$ crystals during heating-cooling cycles from $200 \pm 50^{\circ} \mathrm{C}$ to room temperature in ambient conditions. (a) Straight twin domains appear and are repeatable for subsequent thermal cycles as shown in the second (b) and third (c) heating cycles. Those twins remain after (d) 5 min and (e) 10 min. Twins disappear after (f) $13 \mathrm{~min}$ (below $120^{\circ} \mathrm{C}$ ), indicating a first-order nature of $T_{\mathrm{c} 2}$. Scale bar: $200 \mu \mathrm{m}$.

Next, we discuss the $\mathrm{O}_{T \mathrm{c} 2}^{\prime}$ domains of in situ DF-TEM images [Figs. 5(b), 5(c) and 12]. We name the light-gray area region (i) and dark-gray area region (ii) in Fig. 5(b). First, both light-gray (region i) and dark-gray (region ii) are nucleated $\mathrm{O}_{T \mathrm{c} 2}^{\prime}$ orthorhombic domains above $T_{c 2}$. Figure 12 illustrates the diffraction patterns of regions (i) and (ii) to reveal the $90^{\circ}$-crystallographic-twin relation of regions (i) and (ii). The domain contrast is mainly attributed from the diffraction contrast. Therefore, a reversed contrast of Fig. 5(b) can be obtained by selecting the $S_{3}$ spot as shown in Fig. 5(c). Since the $S_{1}$ spot is allowed only in region (i), we interpret the "brighter" regions of Figs. 5(b) and 5(c), i.e., light-gray domains and white walls, directly.

Given Fig. 5(b) as an example, in the DF-TEM technique, the aperture simultaneously collects the brighter $S_{1}$ spot for the white $\mathrm{O}^{\prime}$ walls and the darker $\mathrm{S}_{1}$ spot for the light-gray $\mathrm{O}_{T \mathrm{c} 2}^{\prime}$ domains. This produces a corresponding contrast difference between them. We find that the $S_{1}$ spot is higher in intensity with the $\mathrm{O}^{\prime}$ structure of a small titling angle (Fig. 13). We likewise observe this intensity asymmetry by selecting the $S_{3}$ spot for region (ii) in Fig. 5(c). Region (ii) reveals a consistent color level as region (i), i.e., light-gray domains and the white walls in Fig. 5(c). Therefore, we assign the light-gray domain and a white string shown in Fig. 5(c), which correspond to the dark-gray domain and a black string shown in Fig. 5(b) to the $\mathrm{O}_{T \mathrm{c} 2}^{\prime}$ and $\mathrm{O}^{\prime}$ phases, respectively. Our in situ heating DF-TEM, structural factors of different tilting models, and energy landscapes provided in this work all point to a likely scenario of a larger tilt magnitude of nucleated $\mathrm{O}^{\prime}{ }_{T \mathrm{c} 2}$ than the $\mathrm{O}^{\prime}$ phase of original DWs. Our observations call for local probes such as annular bright-field (ABF)-scanning TEM (STEM) to further prove the different tilts of domains and domain walls directly.

\section{APPENDIX D: CRYSTAL SYNTHESIS AND CHARACTERIZATION OF Nd $\mathrm{Ca}_{x} \mathrm{Sr}_{1-x} \mathrm{Fe}_{2} \mathrm{O}_{7}$}

We synthesized high-quality polycrystals and single crystals of $\mathrm{Nd}_{2} \mathrm{Ca}_{x} \mathrm{Sr}_{1-x} \mathrm{Fe}_{2} \mathrm{O}_{7}(x=0,0.3,0.4,0.5,0.55$, and 0.6) and $\mathrm{Tb}_{2} \mathrm{Ca}_{x} \mathrm{Sr}_{1-x} \mathrm{Fe}_{2} \mathrm{O}_{7}(x=0,0.3,0.4,0.5,0.6$, and $0.65)$ using solid-state reactions of $\mathrm{Fe}_{2} \mathrm{O}_{3}\left(\mathrm{~Tb}_{4} \mathrm{O}_{7}\right)$ (Alfa, 99.9\%), $\mathrm{SrCO}_{3}$ (Alfa, 99.99\%), $\mathrm{CaCO}_{3}$ (Alfa, 99.95\%), and $\mathrm{Nd}_{2} \mathrm{O}_{3}$ (Alfa, 99.997\%) and the laser-floating zone technique, respectively. The binary oxides were ground together and pelletized, then heated at $1450^{\circ} \mathrm{C}$ for $50 \mathrm{~h}$ in air and regrinding/resintering for $10 \mathrm{~h}$, followed a $100^{\circ} \mathrm{C} / \mathrm{h}$ cooling rate to room temperature. Figures 7 (a) and 7(b) show the lattice parameters and the compositional dependence of x-ray diffraction (XRD) profiles which were examined by x-ray diffraction with a Philips XPert powder diffractometer and the general structure analysis system (GSAS) program. Composition $x \geqslant 0.6$ leads to multiphases, consisting of $n=1$ (perovskite) and 2 RP phases [gray region in Fig. 7(a)]. Selected-area electron diffraction (SAED) patterns of $\mathrm{Nd}_{2} \mathrm{Ca}_{x} \mathrm{Sr}_{1-x} \mathrm{Fe}_{2} \mathrm{O}_{7}$ single crystals reveal the $\mathrm{T}^{\prime}$ phase all the way to $x=0.5$ [Figs. 14(a)-14(c)]. The $\mathrm{T}^{\prime}-\mathrm{O}^{\prime}$ phase boundary is close to $x=0.5$ since $\mathrm{O}^{\prime}$ (Amam) symmetry was found in some grains of $x=0.5$ poly crystallines as a minority phase [Fig. 14(d)]. The $T_{c 1}$ and $T_{c 2}$ of $x=0$ were determined by TEM observation as discussed in the main text. The $T_{c 1}$ of $x=0.5$ was determined by ex situ t-POM observation as shown in Figs. 7(c) and 7(d). The $T_{c 2}$ transition at $x=0.4$ is also identified from an in situ heating under a polarized optical microscope, from which we found that twin patterns appear only above $200 \pm 50^{\circ} \mathrm{C}$ (Fig. 15). The twin structure is reversible through thermal cycling: even the scale and orientation of the twin domains remain largely intact but also exhibit 


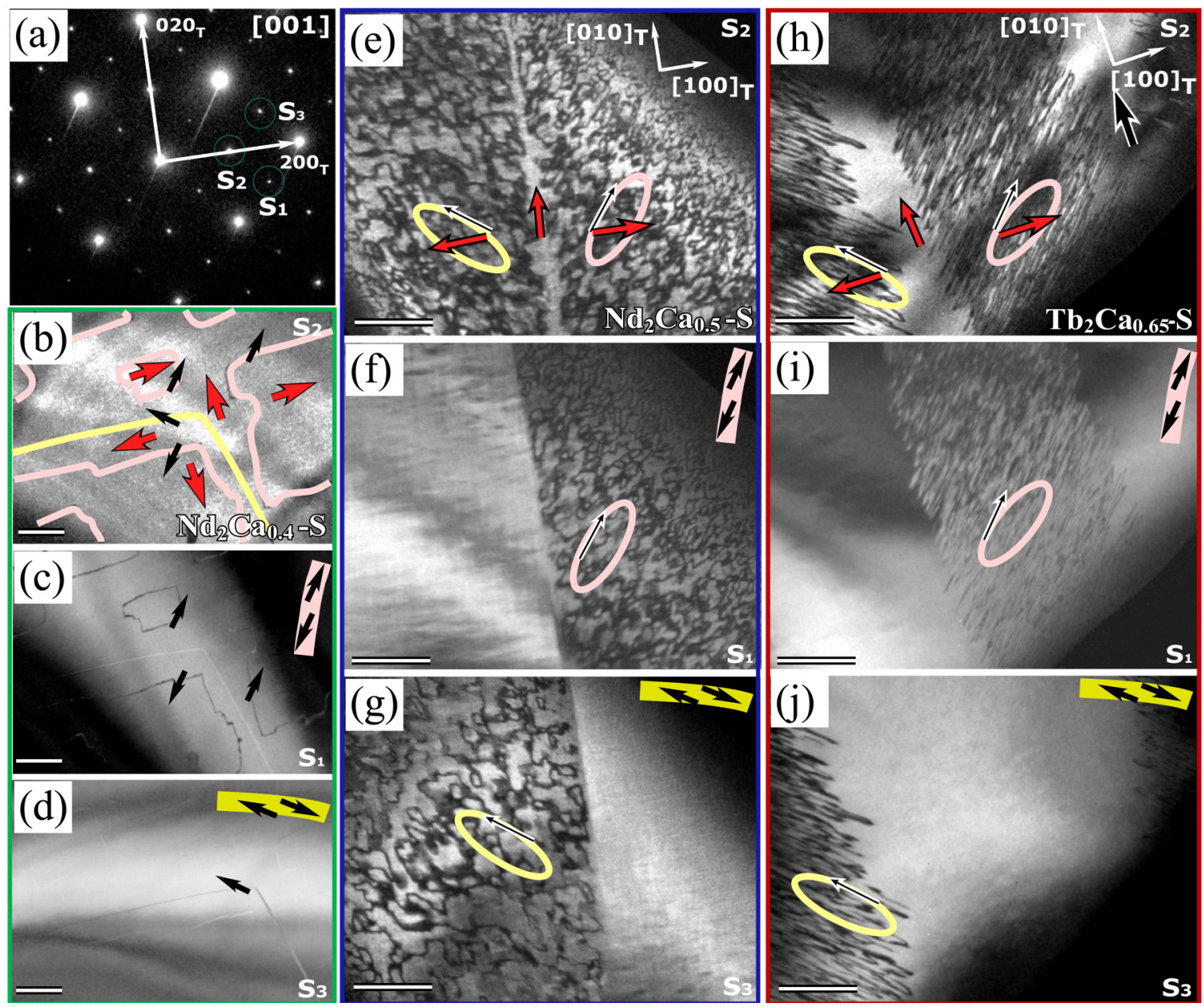

FIG. 16. DF-TEM images showing the nature of $\mathrm{O}^{\prime}$-symmetry of $\mathrm{T}^{\prime} / \mathrm{O}^{\prime}$ loops in slow cooled $\left(100^{\circ} \mathrm{C} / \mathrm{h}\right)$ specimens: $(\mathrm{a})-(\mathrm{d}) \mathrm{Na}_{2} \mathrm{Ca} \mathrm{a}_{0.4}-\mathrm{S}$, (e)- $(\mathrm{g}) \mathrm{Na}_{2} \mathrm{Ca}_{0.5}-\mathrm{S}$, and (h)-(j) $\mathrm{Tb}_{2} \mathrm{Ca}_{0.65}-\mathrm{S}$. Light-red and light-yellow bold lines represent two types of $\mathrm{T}^{\prime} / \mathrm{O}^{\prime}$ loops originated from [110] and $[\overline{1} 10]_{\mathrm{T}}$ tilt axes. Octahedral tilting directions in real space of those dark lines are shown in the upper-right corner. (a) The SAED pattern of $\mathrm{T}^{\prime}\left(P 4_{2} / \mathrm{mnm}\right)$ symmetry along [001] projection with three sets of superlattice peaks: $\mathrm{S}_{1}=1 / 2(30)_{\mathrm{T}}=(120)_{\mathrm{T}^{\prime}}, \mathrm{S}_{2}=1 / 2(200)_{\mathrm{T}}=(110)_{\mathrm{T}^{\prime}}$, and $\mathrm{S}_{3}=1 / 2(310)_{\mathrm{T}}=(210)_{\mathrm{T}^{\prime}}$. (b)-(d) DF-TEM images of $\mathrm{Nd}_{2} \mathrm{Ca}_{0.4}-\mathrm{S}$ via selecting different superlattice peaks for imaging. (c) $\mathrm{S}_{1}-$ and (d) $\mathrm{S}_{3}-$ selected DF-images show a complementary domain-wall map, indicating orthorhombic-like local structure at those walls. (e)-(g) A complete set of superlattice DF-TEM images of $\mathrm{Nd}_{2} \mathrm{Ca}_{0.5}-\mathrm{S}$, showing self-organized $\mathrm{T}^{\prime} / \mathrm{O}^{\prime}$ loops. Light-red and light-yellow oval loops represent $\mathrm{T}^{\prime} / \mathrm{O}^{\prime}$ loops. (h)-(j) A complete set of superlattice DF-TEM images of $\mathrm{Tb}_{2} \mathrm{Ca}_{0.65}-\mathrm{S}$ [Fig. 7(g)]. Scale bar: $0.2 \mu \mathrm{m}$.

a thermal hysteresis, which is the characteristic of first-order phase transitions. As shown in the phase diagram [Fig. 7(a)], $T_{c 1}$ increases a little with increasing $\mathrm{Ca}^{2+}$ concentration while $T_{c 2}$ reveals an opposite trend.

In order to identify the lattice elasticity, we also refined our $\mathrm{x}$-ray data with the orthorhombic structure using the Le Bail method. Our structure analysis shows that $\Delta a / a=(b-a) / a$, where $a$ and $b$ are the $\mathrm{O}^{\prime}$ lattice parameters, varies between 0 and $1.0 \%$, as shown in Figs. 7(a) and 7(b). This variation is attributed to the decrease of the tolerance factor due to the partial chemical substitutions of small $\mathrm{Ca}^{2+}$ for large $\mathrm{Sr}^{2+}$, which leads to an increase of the tilt amplitude. In addition, we also found the lattice spontaneous distortion also varies with different heat treatment as shown in bottom panel of Fig. 7(a). Two different heat treatments of single crystals are discussed: capital S stands for "slowly cooled $\left(100^{\circ} \mathrm{C} / \mathrm{h}\right)$ from 1400 to $1470^{\circ} \mathrm{C}$ (above $T_{c 1}$ )", capital $\mathrm{Q}$ for "quenched from $T_{c 1}$."

\section{APPENDIX E: MICROSTRUCTURES OF $x=0.4$ AND 0.5 IN Nd $\mathrm{Ca}_{x} \mathrm{Sr}_{1-x} \mathrm{Fe}_{2} \mathrm{O}_{7}$}

Figure 16(a) shows the SAED pattern along [001] and three Bragg spots for superlattice DF imaging (a) $\mathrm{S}_{2}=$ $1 / 2(200)_{\mathrm{T}}=(110)_{\mathrm{T}^{\prime}}$, (b) $S_{1}=1 / 2(3 \overline{1} 0)_{\mathrm{T}}=(120)_{\mathrm{T}^{\prime}}$, and $(\mathrm{c})$ $\mathrm{S}_{3}=1 / 2(310)_{\mathrm{T}}=(210)_{\mathrm{T}^{\prime}}$. Those curved dark-contrast lines revealed by selecting the $S_{2}$ spot are DWs (Figs. 16(b), 16(e), and 16(h)]. DF-TEM images using either $S_{1}$ or $S_{3}$ spots will reveal only a part of DWs due to an orthorhombic-like local structure [Figs. 16(c)-16(d), 16(f)-16(g), and 16(i)-16(j)], i.e., the $\mathrm{O}^{\prime}$ phase. Five specimens were discussed in this section: $\mathrm{Nd}_{2} \mathrm{Ca}_{0.4}-\mathrm{Q}(0.2 \%), \mathrm{Nd}_{2} \mathrm{Ca}_{0.4}-\mathrm{S}(0.3 \%), \mathrm{Nd}_{2} \mathrm{Ca}_{0.5}-\mathrm{Q}$ $(0.6 \%), \mathrm{Tb}_{2} \mathrm{Ca}_{0.65}-\mathrm{S}(0.7 \%)$, and $\mathrm{Nd}_{2} \mathrm{Ca}_{0.5}-\mathrm{S}$ (1.0\%) representing different spontaneous distortions. Figures 18(a) and 18(b) show a prototypical type II $\mathrm{Z}_{8} \mathrm{~T}^{\prime}$ vortex in $\mathrm{Nd}_{2} \mathrm{Ca}_{0.4}-\mathrm{Q}$ with $0.2 \%$ spontaneous distortion, consisting of four domains (red arrows) and four domain walls (black arrows) with two types of $\mathrm{O}^{\prime}$ domain walls (light-red and light-yellow bold 

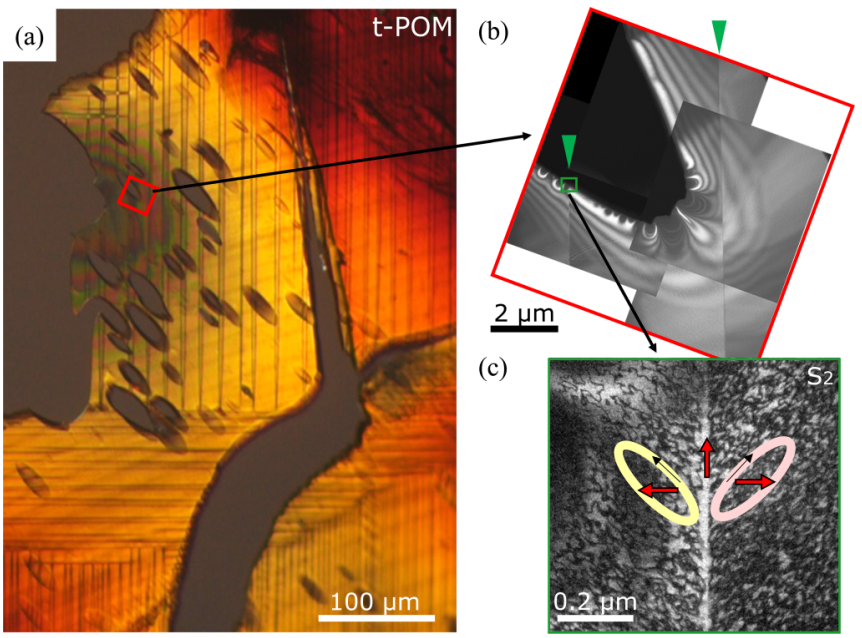

FIG. 17. (a) A polarized optical microscope image of the experimental TEM specimen of $\mathrm{Nd}_{2} \mathrm{Ca}_{0.5}-\mathrm{S}$, showing visible pseudotwins. (b) Low-magnification TEM image of the red-boxed area in (a). Green arrowheads indicate the pseudo-orthorhombic twin boundaries. (c) Magnified DF-TEM image acquired in the green-boxed area shows self-group $\mathrm{T}^{\prime} / \mathrm{O}^{\prime}$ loops.

lines), corresponding to two different tilt axes. As shown in Figs. 16(b)-16(d), the $\mathrm{T}^{\prime} / \mathrm{O}^{\prime}$ loops take place with $0.3 \%$ spontaneous distortion in $\mathrm{Nd}_{2} \mathrm{Ca}_{0.4}-\mathrm{S}$, avoiding any crossing points. Inside and outside a $\mathrm{T}^{\prime} / \mathrm{O}^{\prime}$ loop, the tilt directions of two $\mathrm{T}^{\prime}$ domains always rotate $90^{\circ}$. A drastic change of domain configuration occurs in $\mathrm{Nd}_{2} \mathrm{Ca}_{0.5}-\mathrm{S}$ with $1.0 \%$ spontaneous distortion [Figs. 16(e)-16(g)], even though the matrix with bright contrast is still the $\mathrm{T}^{\prime}$ phase. A similar pattern was found in $\mathrm{Tb}_{2} \mathrm{Ca}_{0.65} \mathrm{Sr}_{0.35} \mathrm{Fe}_{2} \mathrm{O}_{7}$ with $0.7 \%$ spontaneous distortion [Figs. 16(h)-16(j)], where those $\mathrm{T}^{\prime} / \mathrm{O}^{\prime}$ loops tend to sharpen up and avoid mutual intersections. High-density dark wiggled $\mathrm{T}^{\prime} / \mathrm{O}^{\prime}$ loops self-group and elongate along either $[110]_{\mathrm{T}}$ or $[\overline{1} 10]_{\mathrm{T}}$ diagonal direction. The regions with $[110]_{\mathrm{T}}-$ directionelongated loops and those with $[\overline{1} 10]_{\mathrm{T}}$-direction-elongated loops alternate and form a quasiperiodic array over macroscopic distances [Figs. 16(e) and 16(h)]. The origin of these elongated closed loops becomes clear by a complementary domain-wall mapping as shown in Figs. 16(f), 16(g), 16(i) and 16(j). Our DF-TEM images with different superlattice peaks indicate a similar orthorhombic-like local structure of those loops, i.e., the $\mathrm{O}^{\prime}$ symmetry. In addition, the elongated direction is associated with two different tilt axes. Therefore, the orthorhombic twin-like structure observed under a polarized optical microscope is, in fact, associated with a kind of pseudo-orthorhombic twins with the $\mathrm{T}^{\prime}$ matrix and alternating two types of loops as shown in Fig. 17.

The switching between type II $\mathrm{Z}_{8} \mathrm{~T}^{\prime}$ vortex domains and $\mathrm{T}^{\prime} / \mathrm{O}^{\prime}$ loops occurs in $\mathrm{Na}_{2} \mathrm{Ca}_{0.4}$ with a moderate spontaneous
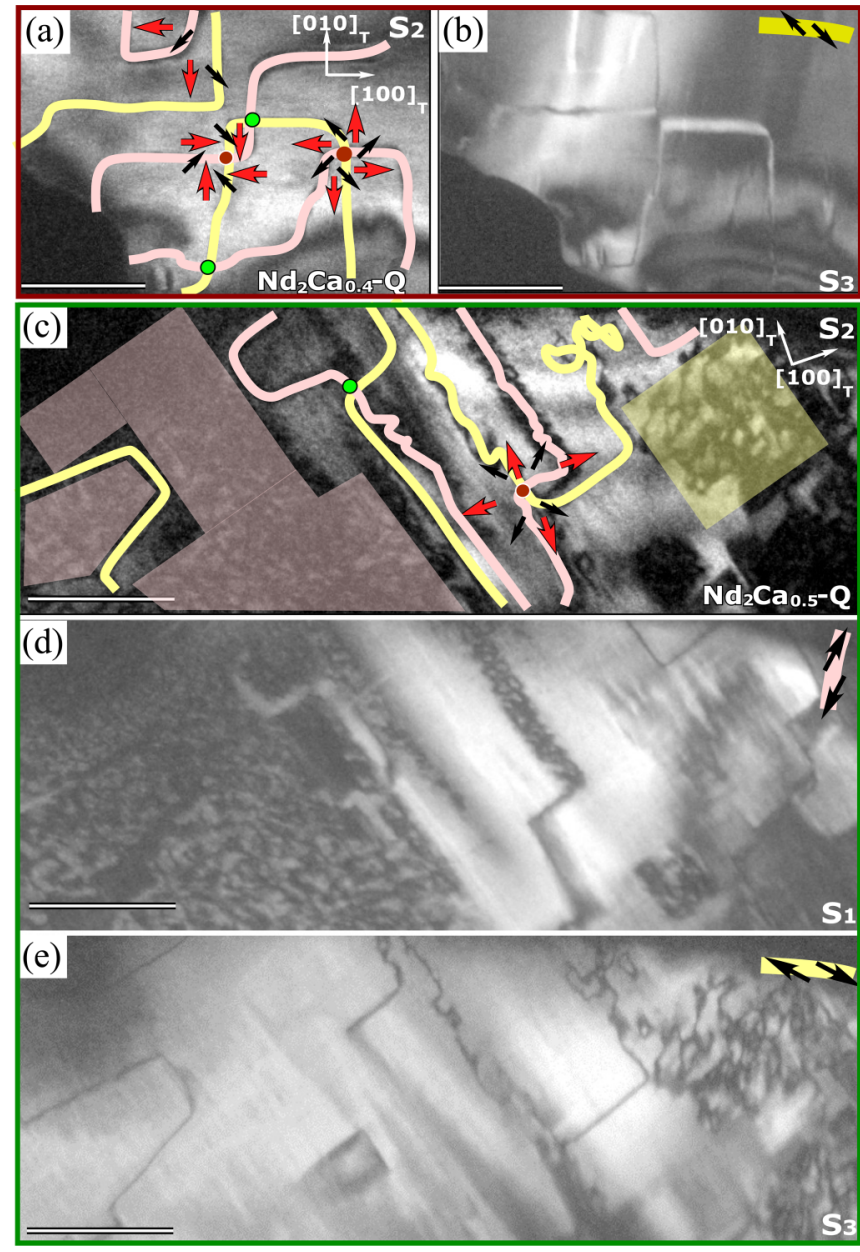

FIG. 18. DF-TEM images showing the nature of $\mathrm{O}^{\prime}$-symmetry of $\mathrm{T}^{\prime} / \mathrm{O}^{\prime}$ loop in quenching specimens: (a), (b) $\mathrm{Na}_{2} \mathrm{Ca}_{0.4}-\mathrm{Q}$ and (c)-(e) $\mathrm{Na}_{2} \mathrm{Ca}_{0.5}$-Q. The assignment is based on DF-images taken using various superlattice spots as explained in Fig. 3. Scale bar: 0.2 $\mu \mathrm{m}$.

distortion. In the case of large spontaneous distortion imposed at $\mathrm{Nd}_{2} \mathrm{Ca}_{0.5}-\mathrm{S}$, a fast cooling across $T_{c 1}$ can restore only a few type II $Z_{8} \mathrm{~T}^{\prime}$ vortex domains [Fig. 18(c)]. Figure 18(c) shows that two types of $\mathrm{T}^{\prime} / \mathrm{O}^{\prime}$ loops tend to form local patches with a very limited intersection, i.e., type II $\mathrm{Z}_{8} \mathrm{~T}^{\prime}$ vortex domains. The size of local patches varies in a wide range from tens of nanometers to submicron. The submicron domains of one type of $\mathrm{T}^{\prime} / \mathrm{O}^{\prime}$ loops still contain small patches of the other type of $\mathrm{T}^{\prime} / \mathrm{O}^{\prime}$ loops. A complete analysis with color-coded is shown in Fig. 18(c). Those local patches can be considered as nanotwins and should behave as invisible under a polarized optical microscope, which explains the reduction of optically observable twin-like structures in Fig. 7(d).
[1] B. P. Abbott et al., (LIGO Scientific Collaboration and Virgo Collaboration), Observation of Gravitational Waves from a Binary Black Hole Merger, Phys. Rev. Lett. 116, 061102 (2016).

[2] V. Ruutu, V. B. Eltsov, A. J. Gill, T. Kibble, M. Krusius, Y. G. Makhlin, B. Placais, G. E. Volovik, and W. Xu, Vortex formation in neutron-irradiated superfluid ${ }^{3} \mathrm{He}$ as an analogue of cosmological defect formation, Nature (London) 382, 334 (1996).

[3] M. M. Salomaa and G. E. Volovik, Quantized vortices in superfluid ${ }^{3} \mathrm{He}$, Rev. Mod. Phys. 59, 533 (1987). 
[4] R. Barboza, U. Bortolozzo, G. Assanto, E. Vidal-Henriquez, M. G. Clerc, and S. Residori, Harnessing Optical Vortex Lattices in Nematic Liquid Crystals, Phys. Rev. Lett. 111, 093902 (2013).

[5] M.-J. Gim, D. A. Beller, and D. K. Yoon, Morphogenesis of liquid crystal topological defects during the nematic-smectic a phase transition, Nat. Commun. 8, 15453 (2017).

[6] N. Balke et al., Enhanced electric conductivity at ferroelectric vortex cores in $\mathrm{BiFeO}_{3}$, Nat. Phys. 8, 81 (2012).

[7] S.-Z. Lin et al., Topological defects as relics of emergent continuous symmetry and Higgs condensation of disorder in ferroelectrics, Nat. Phys. 10, 970 (2014).

[8] T. Choi, Y. Horibe, H. T. Yi, Y. J. Choi, W. Wu, and S. W. Cheong, Insulating interlocked ferroelectric and structural antiphase domain walls in multiferroic $\mathrm{YMnO}_{3}$, Nat. Mater. 9, 253 (2010).

[9] Q. N. Meier et al., Global Formation of Topological Defects in the Multiferroic Hexagonal Manganites, Phys. Rev. X 7, 041014 (2017).

[10] A. K. Yadav et al., Observation of polar vortices in oxide superlattices, Nature (London) 530, 198 (2016).

[11] P. Shafer et al., Emergent chirality in the electric polarization texture of titanate superlattices, Proc. Natl. Acad. Sci. USA 115, 915 (2018)

[12] N. Nagaosa and Y. Tokura, Topological properties and dynamics of magnetic skyrmions, Nat. Nanotechnol. 8, 899 (2013).

[13] A. del Campo and W. H. Zurek, Universality of phase transition dynamics: Topological defects from symmetry breaking, Int. J. Mod. Phys. A 29, 1430018 (2014).

[14] T. W. B. Kibble, Topology of cosmic domains and strings, J. Phys. A: Math. Gen. 9, 1387 (1976).

[15] S. C. Chae, N. Lee, Y. Horibe, M. Tanimura, S. Mori, B. Gao, S. Carr, and S.-W. Cheong, Direct Observation of the Proliferation of Ferroelectric Loop Domains and Vortex-Antivortex Pairs, Phys. Rev. Lett. 108, 167603 (2012).

[16] N. Fowler and D. I. Dierking, Kibble-Zurek scaling during defect formation in a nematic liquid crystal, Chem. Phys. Chem. 18, 812 (2017).

[17] M. J. Bowick, L. Chandar, E. A. Schiff, and A. M. Srivastava, The cosmological kibble mechanism in the laboratory: String formation in liquid crystals, Science 263, 943 (1994).

[18] S. M. Griffin, M. Lilienblum, K. T. Delaney, Y. Kumagai, M. Fiebig, and N. A. Spaldin, Scaling Behavior and Beyond Equilibrium in the Hexagonal Manganites, Phys. Rev. X 2, 041022 (2012).

[19] S. Artyukhin, K. T. Delaney, N. A. Spaldin, and M. Mostovoy, Landau theory of topological defects in multiferroic hexagonal manganites, Nat. Mater. 13, 42 (2013).

[20] S. M. Griffin and N. A. Spaldin, On the relationship between topological and geometric defects, J. Phys. Condens. Matter 29, 343001 (2017).

[21] F.-T. Huang, X. Wang, S. M. Griffin, Y. Kumagai, O. Gindele, M.-W. Chu, Y. Horibe, N. A. Spaldin, and S.-W. Cheong, Duality of Topological Defects in Hexagonal Manganites, Phys. Rev. Lett. 113, 267602 (2014).

[22] M. E. Holtz, K. Shapovalov, J. A. Mundy, C. S. Chang, Z. Yan, E. Bourret, D. A. Muller, D. Meier, and A. Cano, Topological defects in hexagonal manganites: Inner structure and emergent electrostatics, Nano Lett. 17, 5883 (2017).

[23] J. Li, F.-K. Chiang, Z. Chen, C. Ma, M.-W. Chu, C.-H. Chen, H. Tian, H. Yang, and J. Li, Homotopy-theoretic study \& atomic- scale observation of vortex domains in hexagonal manganites, Sci. Rep. 6, 28047 (2016).

[24] F. Xue, N. Wang, X. Wang, Y. Ji, S.-W. Cheong, and L.-Q. Chen, Topological dynamics of vortex-line networks in hexagonal manganites, Phys. Rev. B 97, 020101(R) (2018).

[25] M.-G. Han, Y. Zhu, L. Wu, T. Aoki, V. Volkov, X. Wang, S. C. Chae, Y. S. Oh, and S.-W. Cheong, Ferroelectric switching dynamics of topological vortex domains in a hexagonal manganite, Adv. Mater. 25, 2415 (2013).

[26] P. J. Hickey, Structural studies of layered transition metal oxides, Ph.D. thesis, University of Southampton, 2009.

[27] D. Samaras, A. Collomb, J. C. Joubert, and E. E. F. Bertaut, Etude magnétique du composé $\mathrm{SrTb}_{2} \mathrm{Fe}_{2} \mathrm{O}_{7}$, détermination des structures magnétiques par diffraction neutronique, J. Solid State Chem. 12, 127 (1975).

[28] D. Samaras and R. Chevalier, Etude par spectroscopie Mössbauer d'une serie de ferrites mixyes de terre rare et d'alcalonoterreux: $\operatorname{SrLn}_{2} \mathrm{Fe}_{2} \mathrm{O}_{7}$, J. Magn. Magn. Mater. 5, 35 (1977).

[29] N. N. M. Gurusinghe, J. de la Figuera, J. F. Marco, M. F. Thomas, F. J. Berry, and C. Greaves, Synthesis and characterisation of the $n=2$ Ruddlesden-Popper phases $\mathrm{Ln}_{2} \mathrm{Sr}(\mathrm{Ba}) \mathrm{Fe}_{2} \mathrm{O}_{7}(\mathrm{Ln}=\mathrm{La}, \mathrm{Nd}, \mathrm{Eu})$, Mater. Res. Bull. 48, 3537 (2013).

[30] F.-T. Huang, B. Gao, J. W. Kim, X. Luo, Y. Wang, M.-W Chu, C.-K. Chang, H.-S. Sheu, and S.-W. Cheong, Topological defects at octahedral tilting plethora in bi-layered perovskites, npj Quantum Mater. 1, 16017 (2016).

[31] M. J. Pitcher, P. Mandal, M. S. Dyer, J. Alaria, P. Borisov, H. Niu, J. B. Claridge, and M. J. Rosseinsky, Tilt engineering of spontaneous polarization and magnetization above $300 \mathrm{~K}$ in a bulk layered perovskite, Science 347, 420 (2015).

[32] D. Vanderbilt and M. H. Cohen, Monoclinic and triclinic phases in higher-order Devonshire theory, Phys. Rev. B 63, 094108 (2001).

[33] F.-T. Huang, F. Xue, B. Gao, L. H. Wang, X. Luo, W. Cai, X.-Z Lu, J. M. Rondinelli, L. Q. Chen, and S.-W. Cheong, Domain topology and domain switching kinetics in a hybrid improper ferroelectric, Nat. Commun. 7, 11602 (2016).

[34] L. Chen and J. Shen, Applications of semi-implicit Fourierspectral method to phase field equations, Comput. Phys. Commun. 108, 147 (1998).

[35] D. Orobengoa, C. Capillas, M. I. Aroyo, and J. M. Perez-Mato, AMPLIMODES: Symmetry-mode analysis on the Bilbao crystallographic server, J. Appl. Cryst. 42, 820 (2009).

[36] J. M. Perez-Mato, D. Orobengoa, and M. I. Aroyo, Mode crystallography of distorted structures, Acta. Cryst. A 66, 558 (2010).

[37] V. M. Goldschmidt, Die Gesetze der Krystallochemie, Naturwissenschaften 14, 477 (1926).

[38] See Supplemental Material at http://link.aps.org/supplemental/ 10.1103/PhysRevResearch.3.023216 for phase-field simulation of domain structure evolution starting from two $\mathrm{O}_{T \mathrm{c} 2}^{\prime}$ orthorhombic twin domains.

[39] https://www.cryst.ehu.es/cryst/amplimodes.html.

[40] U. Shmueli, S. R. Hall, and R. W. Grosse-Kunstleve, in International Tables for Crystallography, Vol. B, edited by U. Shmueli (Kluwer Academic Publishers, Dordrecht, 2010), p. 140.

[41] K. Brandenburg, Diamond software, https://www. crystalimpact.com. 